\title{
CONSERVAÇÃO DE SEMENTES DE Myrciaria dubia (H.B.K.) McVAUGH
}

\section{DANIEL FELIPE DE OLIVEIRA GENTIL}

\author{
Tese apresentada à Escola Superior de Agricultura \\ “Luiz de Queiroz", Universidade de São Paulo, \\ para obtenção do título de Doutor em Agronomia. \\ Área de Concentração: Fitotecnia.
}

P I R A C I C A B A

Estado de São Paulo - Brasil

Outubro - 2003 


\title{
CONSERVAÇÃO DE SEMENTES DE Myrciaria dubia (H.B.K.) McVAUGH
}

\section{DANIEL FELIPE DE OLIVEIRA GENTIL}

Engenheiro Agrônomo

Orientador: Prof. Dr. WALTER RODRIGUES DA SILVA

\begin{abstract}
Tese apresentada à Escola Superior de Agricultura
"Luiz de Queiroz", Universidade de São Paulo, para obtenção do título de Doutor em Agronomia. Área de Concentração: Fitotecnia.
\end{abstract}

P I R A C I C A B A

Estado de São Paulo - Brasil

Outubro - 2003 
Dados Internacionais de Catalogação na Publicação (CIP)

DIVISÃO DE BIBLIOTECA E DOCUMENTAÇÃO - ESALQ/USP

Gentil, Da niel Felipe de Oliveira

Conservação de sementes de Myrcia ria dubia (H.B.K) McVaugh /

Daniel Felipe de Oliveira Gentil. - - Piracicaba, 2003.

$41 \mathrm{p}$.

Tese (doutorado) - Escola Supenior de Agricultura Luiz de Queiroz, 2003.

Bibliografia.

1. Amazenamento agrícola 2. Camu-camu 3. Fisiologia vegetal 4. Fruta tropical 5. Fusarium 6. Sementes- Conserva ção 7. Efeito da temperatura 8. Umidade da semente I. Título

CDD 634.6

"Permitida a cópia total ou parcial deste documento, desde que citada a fonte - $\mathrm{O}$ autor" 


\section{AGRADECIMENTOS}

A Deus, pela minha vida e pelas oportunidades que venho tendo na minha jornada profissional.

A Pedro F. Gentil, Edna B. M. de Brito e Sérgio I. G. Braga, pelo estímulo e compreensão durante a realização do Curso.

Aos professores da Escola Superior de Agricultura "Luiz de Queiroz" (ESALQ), especialmente ao meu orientador Walter R. da Silva, pela dedicação com que me transmitiram seus ensinamentos.

Às pesquisadoras do Instituto Nacional de Pesquisas da Amazônia (INPA) Isolde D. K. Ferraz e Rosalee A. Coelho Netto, que gentilmente cederam seus laboratórios para a realização de parte da pesquisa.

Ao pesquisador Sidney A. N. Ferreira (INPA) que, desde a Iniciação Científica, vem contribuindo para o meu aprimoramento científico.

A Salvador B. Torres, Roseli F. Caseiro e Eduardo Suguino, pessoas extraordinárias que têm o dom da solidariedade.

À Coordenação de Aperfeiçoamento do Pessoal de Ensino Superior (CAPES) e à Coordenação de Pós-graduação em Fitotecnia, pela concessão da Bolsa de Doutorado. 


\section{SUMÁRIO}

Página

LISTA DE TABELAS ……………………................................................ vi

RESUMO ..................................................................................... viii

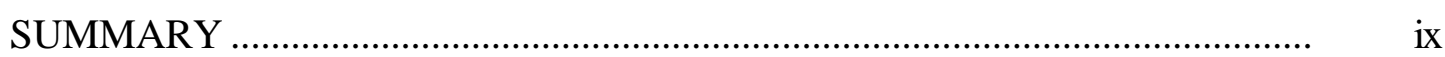

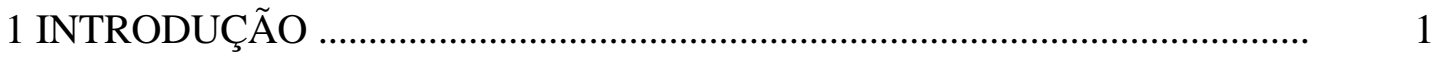

2 REVISÃO DE LITERATURA ................................................................... 4

3 MATERIAL E MÉTODOS ..................................................................... 10

3.1 Colheita dos frutos ................................................................................ 10

3.2 Extração e preparação das sementes .............................................................. 10

3.3 Secagem das sementes ....................................................................... 10

3.4 Armazenamento das sementes .................................................................. 11

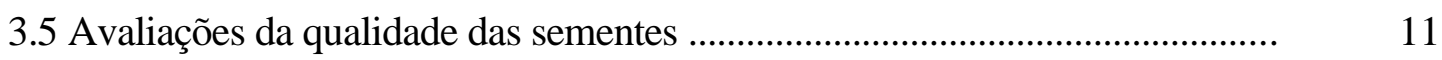

3.5.1 Grau de umidade .................................................................................. 11

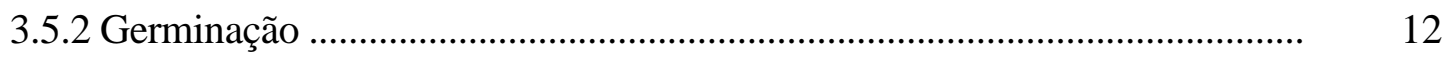

3.5.3 Velocidade de emergência das plântulas .................................................... 12

3.5.4 Emergência das plântulas .............................................................................. 12

3.5.5 Comprimento da plântula ......................................................................... 12

3.5.6 Diâmetro do colo da plântula ........................................................................... 12

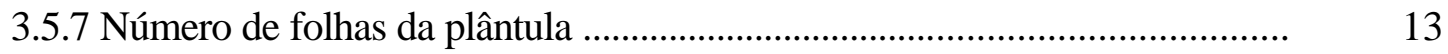

3.5.8 Massa da matéria seca da plântula ................................................................... 13

3.5.9 Sanidade ............................................................................................. 13

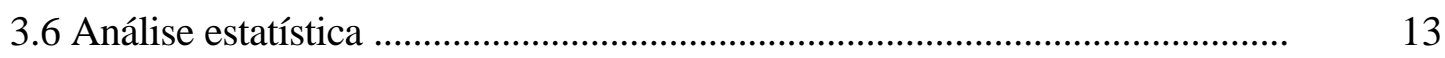

4 RESULTADOS E DISCUSSÃO ............................................................. 15

4.1 Grau de umidade durante o armazenamento ..................................................... 16 


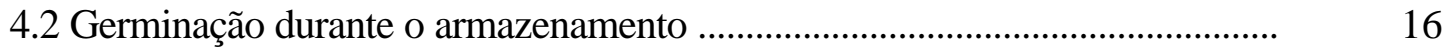

4.3 Vigor durante o armazenamento .................................................................... 17

4.4 Sanidade durante o armazenamento ................................................................ 19

4.5 Considerações gerais ........................................................................................ 20

5 CONCLUSÕES …………....................................................................... 33

REFERÊNCIAS BIBLIOGRÁFICAS _.......................................................... 34 


\section{LISTAS DE TABELAS}

Página

1 Esquema da análise de variância para as avaliações realizadas antes do armazenamento

2 Esquema da análise de variância para as avaliações realizadas em cada período de armazenamento

3 Grau de umidade $(\mathrm{U})$, germinação $(\mathrm{G})$, emergência das plântulas $(\mathrm{E})$, índice de velocidade de emergência das plântulas (IVE), comprimento da plântula (CP), diâmetro do colo da plântula (DC), número de folhas da plântula (NF), massa da matéria seca da plântula (MS), incidência de Fusarium sp. (IF), incidência de Penicillium sp. (IP) e incidência de Aspergillus sp. (IA) em sementes de Myrciaria dubia: valores médios obtidos antes do armazenamento

4 Grau de umidade das sementes de Myrciaria dubia: valores médios (\%, Bu) obtidos durante o armazenamento

5 Germinação das sementes de Myrciaria dubia: valores médios (\%) obtidos durante o armazenamento

6 Emergência das plântulas de Myrciaria dubia: valores médios (\%) obtidos durante o armazenamento das sementes

7 Índice de velocidade de emergência das plântulas de Myrciaria dubia: valores médios obtidos durante o armazenamento das sementes

8 Comprimento da plântula de Myrciaria dubia: valores médios (cm) obtidos durante o armazenamento das sementes

9 Diâmetro do colo da plântula de Myrciaria dubia: valores médios (mm) obtidos durante o armazenamento das sementes 
10 Número de folhas da plântula de Myrciaria dubia: valores médios obtidos durante o armazenamento das sementes

11 Massa da matéria seca da plântula de Myrciaria dubia: valores médios (g) obtidos durante o armazenamento das sementes

12 Incidência de Fusarium sp. nas sementes de Myrciaria dubia: valores médios $(\%)$ obtidos durante o armazenamento

13 Incidência de Penicillium sp. nas sementes de Myrciaria dubia: valores médios (\%) obtidos durante o armazenamento

14 Incidência de Aspergillus sp. nas sementes de Myrciaria dubia: valores médios (\%) obtidos durante o armazenamento 


\title{
CONSERVAÇÃO DE SEMENTES DE Myrciaria dubia (H.B.K.) McVAUGH
}

\author{
Autor: DANIEL FELIPE DE OLIVEIRA GENTIL \\ Orientador: Prof. Dr. WALTER RODRIGUES DA SILVA
}

\section{RESUMO}

As sementes de Myrciaria dubia apresentam curta longevidade e demandam a ampliação do conhecimento sobre fatores interferentes na sua conservação. Diante disso, o presente trabalho objetivou verificar as influências do grau de umidade e da temperatura do ambiente na manutenção da qualidade dessas sementes. Para tanto, sementes com 48, 43, 40, 34, 30, 24, 18 e 14\% de água, acondicionadas em sacos de polietileno, foram armazenadas sob temperaturas controladas de $10^{\circ} \mathrm{C}, 20^{\circ} \mathrm{C}$ e $30^{\circ} \mathrm{C}$, durante 280 dias, e submetidas, periodicamente, a avaliações do grau de umidade, da germinação, do vigor e da sanidade. Foi constatado que as sementes têm a conservação favorecida pela associação do grau de umidade de $43 \%$ com a temperatura de armazenamento de $10^{\circ} \mathrm{C}$; sob essa condição, a incidência de Fusarium sp. não prejudica o desempenho fisiológico das sementes. 
CONSERVATION OF Myrciaria dubia (H.B.K.) McVAUGH SEEDS

Author: DANIEL FELIPE DE OLIVEIRA GENTIL Adviser: Prof. Dr. WALTER RODRIGUES DA SILVA

\section{SUMMARY}

Seeds of Myrciaria dubia have a short lifespan and increased knowledge of the factors that interfere with their conservation is required. The aim of this study was to verify influences of moisture content and storage temperature on the maintenance of seed quality. Seeds with 48, 43, 40, 34, 30, 24, 18 and 14\% water were kept in polyethylene bags at $10^{\circ} \mathrm{C}, 20^{\circ} \mathrm{C}$ and $30^{\circ} \mathrm{C}$ for 280 days, and submitted to periodic evaluations of moisture content, germination, vigour and sanity. Seed conservation was favored by the $43 \%$ water and $10^{\circ} \mathrm{C}$ storage temperature association. In this condition, the incidence of Fusarium sp. does not hamper the seed physiological performance. 


\section{INTRODUÇÃO}

O camu-camu (Myrciaria dubia (H.B.K.) McVaugh), também conhecido como camocamo (Gutierrez, 1969), caçari, araçá-d’água (Ferreira, 1986), araçá-de-igapó (Mera, 1987), guayabo e guayabito (Castañeda, 1961), é uma mirtácea frutífera nativa da Amazônia (McVaugh, 1958; 1963). Apresenta, como sinonímias botânicas, Psidium dubium H.B.K., M. paraensis Berg., M. caurensis Steyerm. (McVaugh, 1963), Eugenia divaricata Benth., M. phillyraeoides Berg., M. divaricata (Benth.) Berg. (McVaugh, 1969), M. spruceana Berg. (Gutierrez, 1969) e M. riedeliana Berg. (Mera, 1987).

A planta, arbusto ou pequena árvore, pode alcançar até $8 \mathrm{~m}$ de altura (McVaugh, 1958). Possui copa ramificada, frondosa, com ramos delgados, flexíveis, pendentes (Gutierrez, 1969), cilíndricos, glabros, lisos e de coloração marrom-clara ou avermelhada. As raízes são profundas e apresentam muitos pêlos absorventes (Picón et al., 1987). As folhas são simples, opostas, glabras, glandulosas, peninérveas, membranáceas e pecioladas; o limbo é ovado, elíptico ou lanceolado; o ápice é agudo ou gradualmente acuminado; a base é arredondada ou subcuneada; a margem é inteira e ligeiramente ondulada; as nervuras são aplanadas na face superior e levemente proeminentes na inferior; a face superior possui coloração verde-escura lustrosa e a inferior verde-clara (McVaugh, 1958; Castañeda, 1961). A inflorescência, axilar ou supra-axilar, apresenta até quatro flores hermafroditas subsésseis dispostas em dois pares; as quatro sépalas são arredondadas, glandulosas, soldadas, caducas e de coloração verde; as quatro pétalas são ovadas, côncavas, glandulosas, livres e de coloração branca; o ovário é ínfero; os estames são numerosos, finos e de coloração branca. O fruto, baga globosa, mede entre 1 e $3 \mathrm{~cm}$ de diâmetro e pesa entre 6 e 9g; o epicarpo delgado é liso, lustroso, glanduloso e possui coloração púrpura ou vermelha; a polpa sucosa é ácida, 
aromática e de coloração branca. As sementes, cujo número varia de um a quatro por fruto, são reniformes, aplanadas e pubescentes; o peso unitário, variando entre 0,6 e 0,9g, é inversamente proporcional ao número de unidades por fruto (McVaugh, 1958; Gutierrez, 1969; Calzada \& Rodriguez, 1980; Picón et al., 1987).

No ambiente natural, estabelece-se às margens de rios e lagos de águas escuras e ácidas (Picón et al., 1987), em solos inundáveis de textura arenosa ou argilo-siltosa, com pH entre 5 e 6 (Calzada, 1980), e sempre a pleno sol (Peters \& Vasquez, 1987). Forma agrupamentos uniformes diferenciados, predominantemente monoespecíficos, com densidade populacional decrescente a partir da margem (Keel \& Prance, 1979). Durante a cheia dos rios, pode permanecer parcialmente submerso por quatro a cinco meses (Calzada, 1980). Os frutos representam fonte de vitaminas e de sais minerais para espécies da ictiofauna, como o tambaqui (Colossoma macropomum), o pacu (Mylossoma spp.), a matrinchã (Brycon cephalus) e a curimatã (Prochilodus nigricans) que, por sua vez, contribuem para a dispersão das sementes (Mera, 1987; Peters \& Vasquez, 1987).

O fruto, em virtude da elevada acidez da polpa, dificilmente é consumido ao natural na alimentação humana, muito embora possa ser apreciado dessa forma como "tira-gosto" (Ferreira, 1986). O usual é processá-lo visando a elaboração de suco, mas as características de elevada suculência, sabor ácido acentuado, coloração intensa e odor pronunciado (Andrade, 1991) possibilitam empregá-lo na obtenção de produtos agroindustriais, como polpa congelada, suco concentrado, suco liofilizado, néctar (Villachica, 1996), sorvete, geléia e licor (Alvarado, 1969). Adicionalmente, o elevado teor de ácido ascórbico, entre 1.420 e $3.133 \mathrm{mg} / 100 \mathrm{~g}$ de polpa (Andrade et al., 1995; Silva \& Andrade, 1997), gera interesse da indústria farmacêutica para a produção de concentrado de vitamina C (Villachica, 1996).

A produção comercial de frutos é, usualmente, oriunda da coleta em populações nativas distribuídas em zonas dos rios Ucayali, Nanay e Napo, no Peru (Villachica \& Labarte, 1998), e Negro e Uatumã, no Brasil. Quando cultivado, tem demonstrado adaptação em solos ácidos, bem drenados e de baixa fertilidade, e sob regimes pluviais com até três meses de estiagem (Villachica et al., 1995), apesar de expressar eventuais alterações fenológicas e diminuição da produção (Picón et al., 1987). 
A propagação, embora possa ser conduzida vegetativamente (Calzada \& Rodriguez, 1980; Enciso \& Villachica, 1993; Ferreira \& Gentil, 1997; Santana, 1998; Suguino, 2002), é rotineiramente realizada por via sexuada. Todavia, em decorrência da curta longevidade das sementes (Calzada \& Rodriguez, 1980), o período de semeadura é restrito. A dificuldade de conservação das sementes concorre para a instabilidade na produção de mudas e para a desestabilização dos estoques em bancos de germoplasma.

A sensibilidade à dessecação é admitida como interferente na conservação dessas sementes (Gentil \& Ferreira, 2000). Assim, tem sido sugerida a manutenção do grau de umidade, em níveis elevados, por intermédio de armazenamento em água (Calzada, 1980; Enciso \& Villachica, 1993) ou em embalagem de polietileno a $20^{\circ} \mathrm{C}$ após breve secagem natural à sombra (Gentil \& Ferreira, 1991).

As informações disponíveis, contudo, não são suficientemente detalhadas para a definição de tecnologias capazes de ampliar o período de conservação dessas sementes. Diante disso, o presente trabalho objetivou verificar as influências do grau de umidade e da temperatura do ambiente na sua conservação. 


\section{REVISÃO DE LITERATURA}

A deterioração de sementes é inevitável e variável entre espécies, entre lotes da mesma espécie e entre unidades do mesmo lote (Delouche, 1968; Harrington, 1972). Embora haja dificuldade na diferenciação entre causas e consequiências desse processo (Marcos Filho, 1999), a seqüência provável de eventos, durante a deterioração, envolve a degeneração das membranas celulares, a danificação dos mecanismos de produção energética e de biossíntese, a redução na velocidade de germinação, a redução do potencial de armazenamento, a desuniformidade e o retardamento do crescimento e do desenvolvimento das plântulas, o aumento na sensibilidade a adversidades ambientais, a redução da emergência de plântulas no campo, o aumento da ocorrência de plântulas anormais e a morte (Delouche \& Baskin,1973).

A deterioração determina o potencial de conservação das sementes (Toledo \& Marcos Filho, 1977). No armazenamento, relaciona-se com a longevidade, com a qualidade inicial das sementes e com as condições do ambiente; como a longevidade é fundamentalmente genética, somente a qualidade inicial das sementes e as condições do ambiente de armazenamento poderão ser manipuladas (Popinigis, 1977).

A longevidade corresponde ao período em que, potencialmente, a semente permanece viável (Toledo \& Marcos Filho, 1977). Uma das primeiras classificações segundo a longevidade, estabelecida por Ewart em 1908 (Hong et al., 1996), dividiu as sementes em microbióticas (longevidade inferior a 3 anos), mesobióticas (longevidade de 3 a 15 anos) e macrobióticas (longevidade superior a 15 anos). Mais recentemente, Harrington (1972) classificou as sementes como sendo de vida curta (longevidade inferior a 10 anos) e longevas (longevidade igual ou superior a 10 anos). Contudo, a expressiva variação na longevidade, entre as espécies enquadradas no mesmo grupo, 
dificulta uma classificação precisa (Popinigis, 1977).

No ambiente de armazenamento, a conservação das sementes está relacionada ao seu grau de umidade, à temperatura e à disponibilidade de oxigênio. Geralmente, é favorecida pela diminuição da atividade metabólica decorrente de reduções no grau de umidade, na temperatura, na umidade relativa e na concentração de oxigênio (Roberts, 1972).

A capacidade das sementes em tolerar a dessecação, entretanto, é variável entre as espécies. A maior parte possui sementes, denominadas ortodoxas, que suportam dessecação a graus de umidade em torno de 5\%; outras possuem sementes, chamadas de intermediárias, que toleram dessecação até graus de umidade de 10 a 12,5\%; finalmente, outras possuem sementes, conhecidas como recalcitrantes, que não toleram dessecação até graus de umidade de 15 a 20\% (Roberts, 1973; Hong \& Ellis, 1996). Recentemente, os conceitos de semente ortodoxa, intermediária e recalcitrante, que até então apresentavam uma abordagem qualitativa, passaram a ter um enfoque quantitativo (Walters, 2000), levando em consideração a magnitude da tolerância à dessecação entre as sementes altamente sensíveis e as que suportam a perda da maior parte da água presente (Berjak \& Pammenter, 2000).

A água, além de conferir estabilidade estrutural às membranas e às proteínas, participa ativamente dos processos metabólicos. Quando é removida, abaixo do limite suportado pela célula, podem ocorrer o aumento da concentração dos solutos, a alteração do pH da solução intracelular, a aceleração de reações degenerativas, a desnaturação de proteínas e a perda da integridade das membranas (Sun \& Leopold, 1997).

Alguns mecanismos de proteção têm sido associados à aquisição de tolerância à dessecação e decorrente manutenção da viabilidade durante o armazenamento: a atuação de sistemas antioxidantes, a concentração e ação de moléculas de proteção, incluindo LEA proteínas ("late embryogenesis abundant proteins"), sacarose e oligossacarídeos (rafinose e estaquiose), a formação do estado vítreo e a operação de mecanismos de reparo durante a reidratação (Sun \& Leopold, 1997; Berjak \& Pammenter, 2000; Hoekstra et al., 2001). Teoricamente, a tolerância à dessecação cresce com o aumento do número de mecanismos de proteção em atividade de tal modo que, provavelmente, 
exista um gradiente de tolerância dependente da interação efetiva entre os mecanismos que estão em funcionamento (Berjak \& Pammenter, 2000).

O comportamento das sementes no armazenamento, fixadas as condições ambientais, é função do grau de umidade apresentado. Nas sementes ortodoxas, tem sido observado que, entre os limites inferior (4 a 7\% de água) e superior (15 a 20\% de água), existe relação negativa entre o grau de umidade e o período de conservação; porém, a secagem abaixo de 4 a 7\% não aumenta, necessariamente, o período de conservação (Roberts \& Ellis, 1989; Ellis, 1991; Probert \& Smith, 1996). Nas sementes intermediárias, a relação entre o grau de umidade e o período de conservação é, possivelmente, semelhante à verificada nas sementes ortodoxas (Ellis, 1991; Gentil, 2001); nesse caso, contudo, o limite inferior de umidade está situado entre 10 e 12,5\% (Hong \& Ellis, 1996).

Acima de 15 a $20 \%$ de água, o comportamento das sementes no armazenamento depende, além do grau de umidade, da disponibilidade de oxigênio. Desse modo, sob condições anaeróbicas, o período de conservação em sementes ortodoxas declina com o aumento do grau de umidade enquanto, sob condições aeróbicas, o período de conservação tende a elevar-se (Roberts \& Ellis, 1989; Probert \& Smith, 1996); isso ocorre porque, embora a deterioração progrida com a elevação do grau de umidade, os mecanismos celulares de reparo acionados são mantidos pelo metabolismo durante a respiração aeróbica (Ibrahim \& Roberts, 1983; Ibrahim et al., 1983). As sementes intermediárias apresentam, provavelmente, comportamento similar ao das ortodoxas (Ellis, 1991; Gentil, 2001). Nas sementes recalcitrantes, embora haja indicações de que o aumento do grau de umidade resulte no aumento do período de conservação sob condições aeróbicas (Pammenter \& Berjak, 1999), uma sutil diminuição do grau de umidade inicial pode, em algumas espécies, prolongar esse período (King \& Roberts, 1982; Hor et al., 1984; Queiroz \& Cavalcante, 1986; Chin, 1988; Boyce, 1989; Bilia et al., 1998).

$\mathrm{Na}$ dessecação de sementes recalcitrantes, que toleram breve secagem, devem ser considerados o grau de umidade de segurança, o grau de umidade crítico e o grau de umidade letal para cada espécie: o grau de umidade de segurança corresponde ao 
conteúdo de água que poderá ser atingido, com a secagem, sem prejuízos à viabilidade das sementes (Hong \& Ellis, 1992); o grau de umidade crítico refere-se ao teor de água no qual é detectado o início da perda da viabilidade (Andrade \& Cunha, 1996); o grau de umidade letal equivale ao valor a partir do qual todas as sementes perdem a viabilidade (Hong \& Ellis, 1992). Portanto, o conhecimento dos graus de umidade de segurança, crítico e letal é indispensável na operação de secagem (Martins et al., 1999).

Em virtude da sensibilidade à dessecação, as sementes recalcitrantes vêm sendo armazenadas, geralmente, sob condição úmida (Evans, 1950; Simão, 1959; Chin, 1978; Farrant et al., 1989). Uma das técnicas utilizadas consiste na estratificação das sementes em substrato higroscópico umedecido como, por exemplo, areia, serragem, vermiculita e pó de carvão vegetal. Todavia, têm sido observados o expressivo aumento no volume do material a ser armazenado, a germinação indesejada e a contaminação por microrganismos (Cardoso et al., 1966; Barrueto et al., 1986; Boyce, 1989; Xia et al., 1992; Ferraz \& Sampaio, 1996; Barbedo, 1997). Assim, principalmente no armazenamento de sementes que germinam rapidamente, o método torna-se ineficiente (Boyce, 1989).

A germinação pode representar perdas significativas em sementes recalcitrantes armazenadas úmidas sob temperaturas superiores a $0^{\circ} \mathrm{C}$ (King \& Roberts, 1979; Chin, 1988). A dormência, fenômeno que poderia evitar essa ocorrência, acha-se ausente em muitas espécies que, dessa maneira, demandam o desenvolvimento de métodos capazes de suprimir a germinação (King \& Roberts, 1979). O uso de substâncias inibidoras, como o ABA (Goldbach, 1979), o emprego de soluções osmóticas (Mumford \& Brett, 1982) e a breve secagem ( $\mathrm{Fu}$ et al., 1990; Andrade et al., 1996) podem auxiliar na conservação das sementes de algumas espécies.

A incidência de microrganismos, em sementes com graus de umidade acima de 10 a 13\%, pode comprometer a viabilidade (Harrington, 1972). Diversos fungos, principalmente do gênero Fusarium (Boyce, 1989), têm sido observados em associação com sementes recalcitrantes armazenadas. A ação prejudicial desses microrganismos pode requerer o controle através de fungicidas (Figueiredo, 1986; Chin, 1988; Fu et al., 1990). 
Um método, para prevenir a germinação e a proliferação de microrganismos no armazenamento de sementes recalcitrantes úmidas, é o uso de ambientes com temperaturas de 0 a $5^{\circ} \mathrm{C}$; contudo, particularmente em espécies de origem tropical, a sensibilidade ao frio dificulta a utilização de temperaturas inferiores a $10^{\circ} \mathrm{C}$ (King \& Roberts, 1979).

As razões da sensibilidade das sementes recalcitrantes a temperaturas baixas, embora superiores a $0^{\circ} \mathrm{C}$, não encontram-se devidamente esclarecidas, mas podem estar relacionadas à desnaturação de proteínas e a alterações na estrutura das membranas celulares (Chin, 1988). Por outro lado, os danos por congelamento, sob temperaturas inferiores a $\mathscr{} \mathscr{C}$, estão associados à formação de cristais de gelo em células de sementes armazenadas com graus de umidade acima de 15 a 20\% (Roberts, 1972); pelo fato das sementes recalcitrantes não tolerarem graus de umidade inferiores a 15-20\% (Roberts, 1973; Hong \& Ellis, 1996), o armazenamento sob temperaturas iguais ou inferiores a $0^{\circ} \mathrm{C}$ pode acarretar danos por congelamento (Roberts \& King, 1980).

A temperatura mínima de armazenamento, tolerada pelas sementes recalcitrantes, varia entre as espécies (Chin, 1988), havendo exemplos de $0^{\circ} \mathrm{C}$ (Bacchi, 1961), $5^{\circ} \mathrm{C}$ (Spalding et al., 1976), $10^{\circ} \mathrm{C}$ (Corbineau \& Côme, 1988; Barbedo, 1997; Barbedo et al., 1998; Bilia et al., 1998), $12^{\circ} \mathrm{C}, 15^{\circ} \mathrm{C}$ (Corbineau \& Côme, 1988) e $17^{\circ} \mathrm{C}$ (Hor et al., 1984).

Independentemente de outros fatores, a conservação de sementes recalcitrantes necessita da manutenção do teor de água, em níveis elevados e constantes, durante o armazenamento (King \& Roberts, 1979). Com isso, a escolha da embalagem deve considerar as condições envolvidas no estabelecimento do ponto de equilíbrio higroscópico entre as sementes e a atmosfera circundante. Em atmosferas saturadas de água, o armazenamento a granel ou em embalagem permeável ao vapor d'água pode representar opção adequada; nos demais casos, a embalagem impermeável constitui alternativa que, restringindo a influência da atmosfera externa no ambiente interno, contribui para evitar alterações no grau de umidade das sementes. No entanto, o uso de embalagem hermética, em decorrência da necessidade de existência de trocas gasosas 
entre as sementes e o ambiente externo, não tem sido recomendado (Bonner, 1978). No armazenamento de sementes úmidas, o suprimento de oxigênio, essencial à respiração, promove a obtenção da energia necessária à sustentação dos mecanismos celulares de reparo e, com isso, possibilita a ampliação do período de conservação (King \& Roberts, 1979; Probert \& Smith, 1996); paralelamente, deve ser relevado o efeito prejudicial do acúmulo de gás carbônico à qualidade fisiológica das sementes (Bacchi, 1961; Zink \& Rochelle, 1964; Toledo \& Marcos Filho, 1977).

O emprego da embalagem de polietileno vem apresentando resultados satisfatórios no armazenamento de diversas espécies (Spalding et al., 1976; Barrueto et al., 1986). Conforme Bonner (1978), essa prática, capaz de prevenir a dessecação, é a mais adequada à conservação de sementes recalcitrantes; diante disso, recomenda o uso de películas de polietileno, com espessura de 0,1mm, para permitir trocas gasosas suficientes entre as sementes e o ambiente externo à embalagem. Entretanto, por particularidades de fabricação, a película pode apresentar desuniformidades de espessura e de porosidade em sua extensão, principalmente quando obtida a partir de material reciclado.

O conhecimento disponível, a respeito da conservação de sementes recalcitrantes, não é suficiente para permitir a definição de tecnologias aptas a atender a variabilidade comportamental existente entre as espécies. Assim sendo, particularmente para vegetais em fase de domesticação, é necessária a continuidade dos estudos. 


\section{MATERIAL E MÉTODOS}

O experimento foi conduzido no Laboratório de Sementes da Coordenação de Pesquisas em Ciências Agronômicas do Instituto Nacional de Pesquisas da Amazônia (INPA), em Manaus/AM.

\subsection{Colheita dos frutos}

Os frutos, com o epicarpo apresentando entre 25 e 100\% de coloração vermelha ou púrpura, foram colhidos em área de várzea amazônica, localizada na Estação Experimental do Ariaú - INPA (Iranduba/AM).

\subsection{Extração e preparação das sementes}

As sementes, extraídas mecanicamente em despolpadeira, foram lavadas em água corrente para a retirada dos resíduos de polpa e de casca. Em seguida, foi realizada a catação manual visando a eliminação das sementes danificadas por broca (Conotrachelus dubiae).

\subsection{Secagem das sementes}

Primeiramente, foi retirada uma amostra de sementes para a verificação do grau de umidade inicial do lote. Após essa determinação, foi coletado o material constituinte do tratamento denominado $48 \% \mathrm{H}_{2} \mathrm{O}$. As sementes restantes foram submetidas à secagem, em ambiente controlado $\left(23 \pm 2^{\circ} \mathrm{C}\right.$ e umidade relativa de $\left.47 \pm 3 \%\right)$, visando a obtenção dos tratamentos 43, 40, 34, 30, 24, 18 e 14\% HbO, referentes aos demais graus de umidade desejados. Durante a secagem, as sementes foram distribuídas, em camada monogranular, sobre prateleiras de estantes com fundo provido de tela plástica. 
Os tratamentos foram obtidos através do acompanhamento da perda de peso das sementes durante a secagem. Para isso, amostras de sementes, com pesos iniciais previamente conhecidos, foram acondicionadas em sacos de filó e distribuídas nas prateleiras para pesagens a intervalos regulares. Os pesos finais das amostras, correspondentes a cada um dos graus de umidade desejados, foram previamente determinados através da equação descrita por Cromarty et al. (1985):

$$
\begin{aligned}
& \text { Pf = Pi }(100-\mathrm{Ui}) \times(100-\mathrm{Uf})^{-1} \text {, onde: } \\
& \text { Pf = peso da amostra }(\mathrm{g}) \text { após a secagem; } \\
& \mathrm{Pi}=\text { peso da amostra }(\mathrm{g}) \text { antes da secagem; } \\
& \mathrm{Ui}=\text { grau de umidade }(\%) \text { antes da secagem; } \\
& \mathrm{Uf}=\text { grau de umidade }(\%) \text { desejado após a secagem. }
\end{aligned}
$$

À medida que foram sendo atingidos os graus de umidade próximos aos desejados, amostras foram retiradas, homogeneizadas e divididas em frações que, por sua vez, foram embaladas em sacos de polietileno $(0,1 \mathrm{~mm}$ de espessura) e mantidas, provisoriamente, em ambiente a $20 \pm 2{ }^{\circ} \mathrm{C}$ durante a obtenção dos demais tratamentos.

\subsection{Armazenamento das sementes}

Posteriormente, as amostras, correspondentes aos diferentes graus de umidade, foram distribuídas em ambientes com temperaturas controladas de $10 \pm 2{ }^{\circ} \mathrm{C}, 20 \pm 2{ }^{\circ} \mathrm{Ce}$ $30 \pm 2{ }^{\circ} \mathrm{C}$. No início do armazenamento (caracterização dos tratamentos graus de umidade) e aos 35, 70, 140, 210 e 280 dias, as sementes foram submetidas às avaliações da qualidade.

\subsection{Avaliações da qualidade das sementes}

3.5.1 Grau de umidade: foi determinado através do método de estufa a $105 \pm 3{ }^{\circ} \mathrm{C}$ por 24 horas (Brasil, 1992), utilizando quatro repetições de 10 sementes inteiras. Os 
resultados, expressos em porcentagem, foram calculados com base no peso úmido.

3.5.2 Germinação: foi realizada em câmara de germinação a $30 \pm 2{ }^{\circ} \mathrm{C}$ (Sposito et al., 1998), empregando quatro repetições de 25 sementes distribuídas sobre duas folhas de papel mata-borrão e acondicionadas em gerbox. As contagens, feitas semanalmente entre a instalação do teste e a estabilização da germinação na população, constituíram os resultados expressos em porcentagem de sementes com, no mínimo, 3mm de raiz primária.

3.5.3 Velocidade de emergência das plântulas: foi realizada em caixas de madeira contendo serragem e areia peneiradas (proporção volumétrica de 1:1), utilizando quatro repetições de 25 sementes dispostas a $2 \mathrm{~cm}$ de profundidade no substrato. Na avaliação, realizada a cada cinco dias entre a instalação do teste e a estabilização da emergência na população, foram consideradas como emersas as plântulas que apresentavam, no mínimo, 5mm de altura da parte aérea. Para o cálculo do Índice de Velocidade de Emergência (IVE) foi empregada a equação proposta por Maguire (1962).

3.5.4 Emergência das plântulas: foi considerado, para o cálculo da porcentagem, o total de plântulas emersas obtido na avaliação da velocidade de emergência (Nakagawa, 1994).

3.5.5 Comprimento da plântula: no encerramento do teste de velocidade de emergência, foi realizada a medição da distância entre os ápices da raiz primária e do epicótilo das plântulas (Nakagawa, 1994). O comprimento médio $(\mathrm{cm})$ da plântula foi obtido através do quociente entre o somatório das medidas registradas e o número de sementes utilizadas.

3.5.6 Diâmetro do colo da plântula: após a obtenção dos dados de comprimento, foi realizada a medição na região do epicótilo situada ao nível da superfície do substrato (Gentil \& Ferreira, 2000). O diâmetro médio (mm) do colo da plântula foi obtido através 
do quociente entre o somatório das medidas registradas e o número de sementes empregadas.

3.5.7 Número de folhas da plântula: após a obtenção dos dados de diâmetro do colo, foi realizada a contagem das folhas das plântulas (Gentil \& Ferreira, 2000). O número médio de folhas da plântula foi obtido através do quociente entre o somatório dos valores registrados e o número de sementes utilizadas.

3.5.8 Massa da matéria seca da plântula: após obtenção dos dados de número de folhas, as plântulas, com os cotilédones extraídos, foram acondicionadas em saco de papel e colocadas em estufa a $80^{\circ} \mathrm{C}$ por 48 horas, previamente às pesagens (Gentil \& Ferreira, 2000). A massa média da matéria seca (g) da plântula foi obtida através do quociente entre a massa total registrada e o número de sementes empregadas.

3.5.9 Sanidade: foram empregadas cinco repetições de 10 sementes distribuídas, em placas de Petri, sobre três folhas de papel de filtro umedecidas com água destilada. A incubação foi realizada em câmara a $25 \pm 2{ }^{\circ} \mathrm{C}$, sob regime alternado de $12 \mathrm{~h}$ de luz/12 h de escuro (Bitencourt \& Homechin, 1998), com lâmpada fluorescente, durante 7 dias. Após esse período, foi efetuada a avaliação da incidência de Fusarium sp., de Penicillium sp. e de Aspergillus sp. com o auxílio de microscópios estereoscópico e composto. Os resultados foram expressos em porcentagem.

\subsection{Análise estatística}

O delineamento experimental adotado foi o inteiramente casualizado, com 8 tratamentos (graus de umidade) no início do armazenamento (Tabela 1) e 24 tratamentos (8 graus de umidade x 3 condições térmicas), em cada época de avaliação, durante o armazenamento (Tabela 2). Na análise estatística, os dados de germinação, de emergência das plântulas e de sanidade das sementes foram transformados em arco seno da raiz quadrada de $\mathrm{x} / 100$; os de número de folhas da plântula foram transformados em raiz quadrada de x (Ferreira, 1991). 
A comparação das médias foi realizada pelo teste de Tukey ao nível de $5 \%$ de probabilidade (Zonta \& Machado, 1984). Os dados de grau de umidade não foram submetidos à análise estatística.

Tabela 1. Esquema da análise de variância para as avaliações realizadas antes do armazenamento

\begin{tabular}{lcc}
\hline CAUSAS DE VARIAÇÃO & \multicolumn{2}{c}{ GRAUS DE LIBERDADE } \\
& Avaliação da sanidade & Demais avaliações \\
\hline Tratamentos & 7 & 7 \\
Resíduo & 32 & 24 \\
Total & 39 & 31 \\
\hline
\end{tabular}

Tabela 2. Esquema da análise de variância para as avaliações realizadas em cada período de armazenamento

\begin{tabular}{lcc}
\hline CAUSAS DE VARIAÇAO & \multicolumn{2}{c}{ GRAUS DE LIBERDADE } \\
& Avaliação da sanidade & Demais avaliações \\
\hline Tratamentos & 23 & 23 \\
Resíduo & 96 & 72 \\
Total & 119 & 95 \\
\hline
\end{tabular}




\section{RESULTADOS E DISCUSSÃO}

As avaliações do grau de umidade, realizadas antes do armazenamento (Tabela 3), indicaram proximidade dos valores experimentalmente atingidos com os desejados e, assim, sugeriram a existência de eficiência no método de acompanhamento da secagem adotado para a obtenção dos tratamentos.

A redução do teor de água, de 48 para 43\%, não afetou imediatamente o desempenho fisiológico das sementes; porém, abaixo de 43\%, o progresso da dessecação tendeu a agravar prejuízos de tal forma que, a partir de 18\%, o desempenho foi anulado (Tabela 3). Gentil \& Ferreira (1991 e 2000), da mesma maneira, constataram evidências de que a dessecação, a valores de 37 e $40 \%$ de água, afeta negativamente a germinação dessas sementes; assim, é provável que o grau crítico de umidade, a partir do qual é detectado o início da perda de viabilidade, esteja situado próximo a 40\%. Paralelamente, foi observado que as sementes não toleraram a dessecação a valores iguais ou inferiores a $18 \%$ de água, muito embora a germinação já tenha sido inexpressiva a 24\%; esse limite de $18 \%$, similar ao de $19 \%$ verificado por Gentil \& Ferreira (2000), mostrou-se letal para as sementes da espécie.

A presença de fungos, anteriormente ao armazenamento (Tabela 3), foi mais evidente nas sementes com graus de umidade inferiores a $34 \%$. Houve a tendência do Fusarium sp. ocorrer em maior número de tratamentos do que o Penicillium sp. e o Aspergillus sp. Em sementes recém-colhidas, geralmente, a incidência de microrganismos de campo, como o Fusarium sp., é maior do que a de microrganismos de armazenamento, como o Penicillium sp. e o Aspergillus sp. (Menten, 1988). No entanto, não foi possível estabelecer relação quantitativamente consistente entre a redução da umidade das sementes e a incidência desses microrganismos, embora o seu 
estabelecimento tenha sido ampliado, nos graus de umidade inferiores ao crítico, paralelamente àredução da qualidade fisiológica.

\subsection{Grau de umidade durante o armazenamento}

A determinação do grau de umidade das sementes, durante o armazenamento (Tabela 4), permitiu verificar a manutenção, experimentalmente aceitável, da identidade dos tratamentos relativos aos graus de umidade nas temperaturas consideradas.

\subsection{Germinação durante o armazenamento}

Os dados obtidos (Tabela 5) indicaram que a emperatura de $30^{\circ} \mathrm{C}$ foi a que anulou, no menor período (140 dias), a germinação das sementes de todos os graus de umidade; as sementes com $43 \%$ de água, embora apresentando a maior porcentagem de germinação observada nessa temperatura, tiveram deterioração acentuada após os 35 dias de armazenamento.

No ambiente a $20^{\circ} \mathrm{C}$, as sementes com graus de umidade de 48 e $43 \%$ destacaram-se positivamente das demais; quando comparados entre si, aos 140 dias de armazenamento, $43 \%$ foi o mais favorável à conservação das sementes, muito embora tenha apresentado germinação nula no período seguinte (210 dias). Os valores obtidos nos demais graus de umidade, apesar de superiores aos observados a $30^{\circ} \mathrm{C}$, expressaram comportamento geral inferior aos verificados em $48 \%$ e, principalmente, $43 \%$ de água; a única particularidade, a ser destacada, foi a das sementes com grau de umidade de $34 \%$ que, de modo exclusivo, não tiveram a germinação anulada no período experimental.

No armazenamento a $10^{\circ} \mathrm{C}$, os graus de umidade de 48 e $43 \%$ favoreceram a conservação; contudo, enquanto $48 \%$ apresentou redução acentuada nos valores a partir de 140 dias, $43 \%$ superou os demais graus de umidade no final do período experimental (210 e 280 dias).

Os resultados revelaram que, fixados os graus de umidade, as sementes tenderam a demonstrar superioridade na germinação à medida que a temperatura de 
armazenamento foi reduzida e, desse modo, $10^{\circ} \mathrm{C}$ mostrou-se mais favorável do que as demais. Em geral, o armazenamento de sementes, com graus de umidade elevados, é favorecido pela adoção de temperaturas inferiores à do ambiente natural de produção (Hor et al., 1984; Corbineau \& Côme, 1988; Villalobos et al., 1992; Andrade et al., 1996; Barbedo, 1997; Bilia et al., 1998); a elevação da temperatura, neste caso, tende a acelerar a deterioração (Harrington, 1972; Roberts, 1972).

Por outro lado, fixadas as temperaturas de armazenamento, os dados obtidos confirmaram os efeitos negativos, observados imediatamente após a dessecação, sobre a germinação. Com o progresso do período de armazenamento, as sementes com graus de umidade inferiores a $43 \%$ tenderam a demonstrar redução acentuada no desempenho; a partir de $24 \%$, os dados foram invariavelmente nulos ou estatisticamente similares aos nulos. Entretanto, no decorrer do período experimental, as sementes com grau de umidade de $43 \%$, que antes do armazenamento tinham apresentado desempenho similar ao das sementes com 48\%, mostraram tendência de superioridade em relação às demais, particularmente sob a temperatura de $10^{\circ} \mathrm{C}$. Esse benefício, decorrente de pequena redução do grau de umidade inicial, tem sido observado em espécies que apresentam sementes sensíveis à dessecação (King \& Roberts, 1982; Hor et al., 1984; Queiroz \& Cavalcante, 1986; Chin, 1988; Boyce, 1989; Bilia et al., 1998).

Assim, interpretando o comportamento dos dados durante os 280 dias de armazenamento, verificou-se que a combinação do grau de umidade de $43 \%$ com a temperatura de $10^{\circ} \mathrm{C}$ destacou-se no favorecimento à manutenção do poder germinativo das sementes de camu-camu.

\subsection{Vigor durante o armazenamento}

O vigor das sementes foi estimado pelos testes de emergência, índice de velocidade de emergência, comprimento, diâmetro do colo, número de folhas e massa da matéria seca das plântulas (Tabelas 6, 7, 8, 9, 10 e 11, respectivamente). A interpretação dos dados não identificou variações expressivas entre as indicações fornecidas pelos diferentes testes; desse modo, os tratamentos puderam ser comparados segundo o 
desempenho predominante no conjunto das avaliações.

No ambiente a $30^{\circ} \mathrm{C}$, a redução do vigor foi acentuada em todos os graus de umidade, especialmente nos iguais ou inferiores a 40\%; quando comparados entre si, o de $43 \%$, apesar de anular o desempenho das sementes a partir dos 140 dias de armazenamento, destacou-se positivamente dos demais.

De modo geral, o desempenho observado a $20^{\circ} \mathrm{C}$ foi superior ao verificado a $30^{\circ} \mathrm{C}$. Entre os graus de umidade, $43 \%$ destacou-se ao manter superioridade durante 140 dias de armazenamento; no entanto, similarmente ao observado na germinação, $34 \%$ foi o único em que o vigor deixou de ser anulado durante os 280 dias do período experimental.

No armazenamento a $10^{\circ} \mathrm{C}$, o declínio do vigor foi mais acentuado nas sementes com graus de umidade de 34 e $30 \%$ do que o verificado em 40, 48 e, principalmente, $43 \%$. No final do período experimental (210 e 280 dias), foi evidenciada a superioridade das sementes com grau de umidade de $43 \%$ em relação às demais, independentemente da temperatura considerada.

Os resultados obtidos nos testes de vigor, apresentando tendência geral similar à verificada na germinação, evidenciaram os efeitos da dessecação sobre a qualidade fisiológica das sementes. A dessecação, a graus de umidade iguais ou inferiores a $24 \%$, provocou danos acentuados que corroboram a indicação do comportamento recalcitrante (Gentil \& Ferreira, 2000). As sementes com graus de umidade de 48, 40, 34 e $30 \%$ apresentaram redução do vigor suficiente para, independentemente da temperatura, atingir valores nulos ou próximos aos nulos no final do período de armazenamento. Por outro lado, as sementes com $43 \%$ de água evidenciaram permanente tendência de superioridade no desempenho em relação às demais. A redução do grau de umidade de 48 para 43\%, portanto, contribuiu para a manutenção da qualidade fisiológica das sementes armazenadas.

Paralelamente, ao contrário do observado em outras espécies com sementes sensíveis à dessecação (Hor et al., 1984; Corbineau \& Côme, 1988), a redução da temperatura favoreceu a conservação das sementes de camu-camu. Desse modo, a associação de $10^{\circ} \mathrm{C}$ com o grau de umidade de $43 \%$ demonstrou ser adequada ao 
armazenamento das sementes dessa espécie.

\subsection{Sanidade durante o armazenamento}

A análise de sanidade evidenciou que, em geral, a incidência de Fusarium sp. (Tabela 12) foi favorecida nas sementes com graus de umidade iguais ou superiores a $30 \%$, particularmente sob $30^{\circ} \mathrm{C}$ e $20^{\circ} \mathrm{C}$. As ocorrências de Penicillium sp. (Tabela 13) e de Aspergillus sp. (Tabela 14), ao contrário do observado em Fusarium sp., tenderam a ser intensificadas nas sementes com graus de umidade inferiores a 30\%. Contudo, Penicillium sp. foi mais freqüente do que Aspergillus sp. e, paralelamente, não evidenciou tendência de alteração na incidência com a mudança da temperatura de armazenamento.

O grau de umidade e a temperatura são reconhecidos como fatores interferentes no estabelecimento de fungos em sementes armazenadas (Christensen, 1972). O Fusarium sp. necessita, geralmente, de teor de água superior a 30\% para desenvolver-se (Copeland \& McDonald, 1995), enquanto o Penicillium sp. e o Aspergillus sp. o fazem em graus de umidade situados entre 10 e 20\% (Harrington, 1972). A temperatura ótima para o desenvolvimento desses microrganismos está situada entre $30^{\circ} \mathrm{C}$ e $33^{\circ} \mathrm{C}$, sendo a máxima de $55^{\circ} \mathrm{C}$ e a mínima de $0^{\circ} \mathrm{C}$ (Copeland \& McDonald, 1995).

Com a ampliação do período de armazenamento, a ocorrência de Fusarium sp. tendeu a ser nula em sementes com graus de umidade situados entre 14 e $24 \%$. As incidências de Penicillium sp. e de Aspergillus sp. não indicaram, no entanto, evidências quantitativas relacionadas ao prazo de armazenamento. Comumente, o decréscimo na população de patógenos de campo vem seguido por um acréscimo na população de patógenos de armazenamento (Menten, 1988).

Considerando que o desempenho das sementes foi praticamente anulado, imediatamente após a dessecação abaixo de $30 \%$ de água, não foi observada associação direta entre a qualidade fisiológica e a incidência de microrganismos nas sementes armazenadas com graus de umidade situados entre 14 e $24 \%$. Por outro lado, a ocorrência prioritária de Fusarium sp. nas sementes de desempenho fisiológico superior, 
com graus de umidade situados acima de 30\%, sugere que esse fungo, apesar de importante no armazenamento de sementes recalcitrantes (Boyce, 1989), não deprime a qualidade fisiológica das sementes de camu-camu.

\subsection{Considerações gerais}

A verificação do nível de tolerância à dessecação é procedimento necessário para antever o comportamento das sementes durante o armazenamento (Hong \& Ellis, 1996). Assim, similarmente ao observado por Gentil \& Ferreira (2000), foi constatado que as sementes de camu-camu não toleram dessecação a valores, iguais ou inferiores a $18 \%$ de água, inseridos no intervalo (15 a 20\%) considerado letal para as sementes recalcitrantes (Roberts, 1973; Hong \& Ellis, 1996). Por outro lado, a dessecação de 48 para 43\%, além de não induzir danos imediatos à qualidade fisiológica, favoreceu a conservação das sementes.

Apesar da variação da temperatura não haver interferido apreciavelmente na ocorrência de microrganismos, a de $10^{\circ} \mathrm{C}$, diferentemente do verificado por Gentil \& Ferreira (1991), foi adequada à preservação da qualidade fisiológica das sementes.

Desse modo, foi verificado que a longevidade das sementes de camu-camu, considerada efêmera (Calzada, 1980; Enciso \& Villachica, 1993; Gentil \& Ferreira, 1991), está sujeita a variações expressivas e decorrentes do grau de umidade e da temperatura existentes durante o armazenamento. 
Tabela 3. Grau de umidade (U), germinação (G), emergência das plântulas (E), índice de velocidade de emergência das plântulas (IVE), comprimento da plântula (CP), diâmetro do colo da plântula (DC), número de folhas da plântula (NF), massa da matéria seca da plântula (MS), incidência de Fusarium sp. (IF), incidência de Penicillium sp. (IP)e incidência de Aspergillus sp. (IA) em sementes de Myrciaria dubia: valores médios ${ }^{1}$ obtidos antes do armazenamento

\begin{tabular}{cccccccccccc}
\hline Tratamentos & $\begin{array}{c}\mathrm{U} \\
(\%, \mathrm{Bu})\end{array}$ & $\begin{array}{c}\mathrm{G} \\
(\%)\end{array}$ & $\begin{array}{c}\mathrm{E} \\
(\%)\end{array}$ & $\mathrm{IVE}$ & $\begin{array}{c}\mathrm{CP} \\
(\mathrm{cm})\end{array}$ & $\begin{array}{c}\mathrm{DC} \\
(\mathrm{mm})\end{array}$ & $\begin{array}{c}\mathrm{NF} \\
(\mathrm{g})\end{array}$ & $\begin{array}{c}\mathrm{IF} \\
(\%)\end{array}$ & $\begin{array}{c}\mathrm{IP} \\
(\%)\end{array}$ & $\begin{array}{c}\mathrm{IA} \\
(\%)\end{array}$ \\
\hline $48 \% \mathrm{H}_{2} \mathrm{O}$ & 48,3 & $99 \mathrm{a}$ & $86 \mathrm{ab}$ & $3,258 \mathrm{a}$ & $23,52 \mathrm{a}$ & $1,49 \mathrm{a}$ & $13,04 \mathrm{a}$ & $0,300 \mathrm{a}$ & $0 \mathrm{~d}$ & $0 \mathrm{~d}$ & $0 \mathrm{c}$ \\
$43 \% \mathrm{H}_{2} \mathrm{O}$ & 43,2 & $97 \mathrm{a}$ & $94 \mathrm{a}$ & $3,215 \mathrm{a}$ & $24,34 \mathrm{a}$ & $1,52 \mathrm{a}$ & $12,79 \mathrm{a}$ & $0,273 \mathrm{a}$ & $6 \mathrm{~cd}$ & $0 \mathrm{~d}$ & $0 \mathrm{c}$ \\
$40 \% \mathrm{H}_{2} \mathrm{O}$ & 39,5 & $80 \mathrm{~b}$ & $77 \mathrm{~b}$ & $2,962 \mathrm{a}$ & $17,26 \mathrm{~b}$ & $1,17 \mathrm{~b}$ & $9,02 \mathrm{a}$ & $0,185 \mathrm{~b}$ & $12 \mathrm{~cd}$ & $2 \mathrm{~d}$ & $0 \mathrm{c}$ \\
$34 \% \mathrm{H}_{2} \mathrm{O}$ & 33,9 & $50 \mathrm{c}$ & $37 \mathrm{c}$ & $1,234 \mathrm{~b}$ & $7,02 \mathrm{c}$ & $0,49 \mathrm{c}$ & $3,83 \mathrm{~b}$ & $0,068 \mathrm{c}$ & $20 \mathrm{bc}$ & $0 \mathrm{~d}$ & $0 \mathrm{c}$ \\
$30 \% \mathrm{H}_{2} \mathrm{O}$ & 30,1 & $28 \mathrm{~d}$ & $21 \mathrm{c}$ & $0,602 \mathrm{c}$ & $4,48 \mathrm{c}$ & $0,30 \mathrm{c}$ & $2,43 \mathrm{~b}$ & $0,037 \mathrm{~cd}$ & $44 \mathrm{ab}$ & $6 \mathrm{~d}$ & $6 \mathrm{abc}$ \\
$24 \% \mathrm{H}_{2} \mathrm{O}$ & 23,7 & $4 \mathrm{e}$ & $3 \mathrm{~d}$ & $0,064 \mathrm{~cd}$ & $0,59 \mathrm{~d}$ & $0,04 \mathrm{~d}$ & $0,40 \mathrm{c}$ & $0,006 \mathrm{~d}$ & $10 \mathrm{~cd}$ & $90 \mathrm{a}$ & $2 \mathrm{bc}$ \\
$18 \% \mathrm{H}_{2} \mathrm{O}$ & 18,2 & $0 \mathrm{e}$ & $0 \mathrm{~d}$ & $0,000 \mathrm{~d}$ & $0,00 \mathrm{~d}$ & $0,00 \mathrm{~d}$ & $0,00 \mathrm{c}$ & $0,000 \mathrm{~d}$ & $74 \mathrm{a}$ & $30 \mathrm{c}$ & $12 \mathrm{a}$ \\
$14 \% \mathrm{H}_{2} \mathrm{O}$ & 14,3 & $0 \mathrm{e}$ & $0 \mathrm{~d}$ & $0,000 \mathrm{~d}$ & $0,00 \mathrm{~d}$ & $0,00 \mathrm{~d}$ & $0,00 \mathrm{c}$ & $0,000 \mathrm{~d}$ & $46 \mathrm{ab}$ & $68 \mathrm{~b}$ & $12 \mathrm{a}$ \\
\hline
\end{tabular}

1. Médias seguidas de mesma letra na coluna não diferem entre si, pelo teste de Tukey, ao nível de 5\% de probabilidade. 
Tabela 4. Grau de umidade das sementes de Myrciaria dubia: valores médios (\%, Bu) obtidos durante o armazenamento

\begin{tabular}{|c|c|c|c|c|c|}
\hline \multirow{2}{*}{$\begin{array}{l}\text { Tratamentos (temperatura / } \\
\text { grau de umidade) }\end{array}$} & \multicolumn{5}{|c|}{ Períodos de armazenamento (dias) } \\
\hline & 35 & 70 & 140 & 210 & 280 \\
\hline $30^{\circ} \mathrm{C} / 48 \% \mathrm{HzO}$ & 50,3 & 52,5 & 50,2 & 49,7 & 50,8 \\
\hline $43 \% \mathrm{HbO}$ & 43,2 & 43,8 & 45,6 & 45,2 & 43,1 \\
\hline $40 \% \mathrm{H}$ & 40,1 & 38,3 & 36,5 & 38,3 & 38,9 \\
\hline $34 \% \mathrm{H}$ & 33,3 & 32,9 & 30,4 & 32,2 & 33,9 \\
\hline $30 \% \mathrm{H} 2 \mathrm{O}$ & 29,1 & 30,1 & 30,8 & 27,8 & ND \\
\hline $24 \% \mathrm{H} 2 \mathrm{O}$ & 22,9 & 21,2 & 24,5 & 22,8 & 21,6 \\
\hline $18 \% \mathrm{HzO}$ & 17,6 & 18,5 & 15,7 & 16,3 & 15,2 \\
\hline $14 \% \mathrm{HzO}$ & 13,7 & 13,2 & 12,1 & 13,4 & 12,3 \\
\hline $20^{\circ} \mathrm{C} / 48 \% \mathrm{H} 2 \mathrm{O}$ & 47,5 & 48,9 & 54,0 & 53,2 & 52,8 \\
\hline $43 \% \mathrm{HbO}$ & 42,8 & 43,3 & 42,5 & 47,5 & 46,5 \\
\hline $40 \% \mathrm{H} 2 \mathrm{O}$ & 39,5 & 39,3 & 41,8 & 39,7 & 40,7 \\
\hline $34 \% \mathrm{HzO}$ & 34,4 & 34,8 & 34,2 & 36,6 & 34,8 \\
\hline $30 \% \mathrm{HzO}$ & 29,9 & 30,5 & 30,4 & 30,5 & 30,3 \\
\hline $24 \% \mathrm{H2O}$ & 24,1 & 24,1 & 25,2 & 23,7 & 23,4 \\
\hline $18 \% \mathrm{H} \mathrm{O}$ & 19,1 & 19,1 & 17,8 & 18,1 & 17,3 \\
\hline $14 \% \mathrm{HzO}$ & 14,7 & 14,3 & 14,5 & 13,7 & 13,6 \\
\hline $10^{\circ} \mathrm{C} / 48 \% \mathrm{H} \mathrm{O}$ & 48,0 & 49,0 & 52,2 & 52,8 & 50,0 \\
\hline $43 \% \mathrm{H} O$ & 43,6 & 44,6 & 43,4 & 43,6 & 42,5 \\
\hline $40 \% \mathrm{H} 2 \mathrm{O}$ & 39,0 & 39,8 & 42,3 & 40,2 & 43,4 \\
\hline $34 \% \mathrm{H} 2 \mathrm{O}$ & 34,4 & 32,6 & 35,0 & 34,7 & 35,1 \\
\hline $30 \% \mathrm{H}_{2} \mathrm{O}$ & 29,2 & 31,1 & 30,9 & 29,8 & 30,2 \\
\hline $24 \% \mathrm{HbO}$ & 23,2 & 23,0 & 23,8 & 23,7 & 24,0 \\
\hline $18 \% \mathrm{HbO}$ & 18,3 & 19,1 & 18,9 & 19,0 & 17,2 \\
\hline $14 \% \mathrm{HzO}$ & 14,1 & 13,9 & 14,3 & 14,6 & 14,0 \\
\hline
\end{tabular}

ND: não determinado. 
Tabela 5. Germinação das sementes de Myrciaria dubia: valores médios ${ }^{1}$ (\%) obtidos durante o armazenamento

\begin{tabular}{|c|c|c|c|c|c|c|}
\hline \multirow{2}{*}{\multicolumn{2}{|c|}{$\begin{array}{l}\text { Tratamentos (temperatura / } \\
\text { grau de umidade) }\end{array}$}} & \multicolumn{5}{|c|}{ Períodos de armazenamento (dias) } \\
\hline & & 35 & 70 & 140 & 210 & 280 \\
\hline \multirow[t]{8}{*}{$30^{\circ} \mathrm{C} /$} & $48 \% \mathrm{HzO}$ & $31 \mathrm{de}$ & $0 \mathrm{f}$ & $0 \mathrm{e}$ & $0 \mathrm{~d}$ & $0 \mathrm{c}$ \\
\hline & $43 \% \mathrm{H}_{2} \mathrm{O}$ & 99 a & $17 \mathrm{de}$ & $0 \mathrm{e}$ & $0 \mathrm{~d}$ & $0 \mathrm{c}$ \\
\hline & $40 \% \mathrm{H} 2 \mathrm{O}$ & $0 \mathrm{f}$ & $0 \mathrm{f}$ & $0 \mathrm{e}$ & $0 \mathrm{~d}$ & $0 \mathrm{c}$ \\
\hline & $34 \% \mathrm{H} 2 \mathrm{O}$ & $1 \mathrm{f}$ & $0 \mathrm{f}$ & $0 \mathrm{e}$ & $0 \mathrm{~d}$ & $0 \mathrm{c}$ \\
\hline & $30 \% \mathrm{HzO}$ & $0 \mathrm{f}$ & $0 \mathrm{f}$ & $0 \mathrm{e}$ & $0 \mathrm{~d}$ & $0 \mathrm{c}$ \\
\hline & $24 \% \mathrm{H} 2 \mathrm{O}$ & $2 \mathrm{f}$ & $0 \mathrm{f}$ & $0 \mathrm{e}$ & $0 \mathrm{~d}$ & $0 \mathrm{c}$ \\
\hline & $18 \% \mathrm{H}$ & $0 \mathrm{f}$ & $0 \mathrm{f}$ & $0 \mathrm{e}$ & $0 \mathrm{~d}$ & $0 \mathrm{c}$ \\
\hline & $14 \% \mathrm{H}_{2} \mathrm{O}$ & $0 \mathrm{f}$ & $0 \mathrm{f}$ & $0 \mathrm{e}$ & $0 \mathrm{~d}$ & $0 \mathrm{c}$ \\
\hline \multirow[t]{8}{*}{$20^{\circ} \mathrm{C} /$} & $48 \% \mathrm{H} 2 \mathrm{O}$ & $100 \mathrm{a}$ & $100 \mathrm{a}$ & $0 \mathrm{e}$ & $0 \mathrm{~d}$ & $0 \mathrm{c}$ \\
\hline & $43 \% \mathrm{H}_{2} \mathrm{O}$ & 99 a & $95 \mathrm{a}$ & $97 \mathrm{a}$ & $0 \mathrm{~d}$ & $0 \mathrm{c}$ \\
\hline & $40 \% \mathrm{HzO}$ & $57 \mathrm{c}$ & $39 \mathrm{~b}$ & $57 \mathrm{~b}$ & $0 \mathrm{~d}$ & $0 \mathrm{c}$ \\
\hline & $34 \% \mathrm{HzO}$ & $19 \mathrm{de}$ & 20 cde & $32 \mathrm{c}$ & $22 \mathrm{c}$ & $3 \mathrm{~b}$ \\
\hline & $30 \% \mathrm{H}_{2} \mathrm{O}$ & $18 \mathrm{e}$ & $13 \mathrm{de}$ & $7 d$ & $1 \mathrm{~d}$ & $0 \mathrm{c}$ \\
\hline & $24 \% \mathrm{H} 2 \mathrm{O}$ & $2 \mathrm{f}$ & $0 \mathrm{f}$ & $0 \mathrm{e}$ & $0 \mathrm{~d}$ & $0 \mathrm{c}$ \\
\hline & $18 \% \mathrm{H} 2 \mathrm{O}$ & $0 \mathrm{f}$ & $0 \mathrm{f}$ & $0 \mathrm{e}$ & $0 \mathrm{~d}$ & $0 \mathrm{c}$ \\
\hline & $14 \% \mathrm{HzO}$ & $0 \mathrm{f}$ & $0 \mathrm{f}$ & $0 \mathrm{e}$ & $0 \mathrm{~d}$ & $0 \mathrm{c}$ \\
\hline \multirow[t]{8}{*}{$10^{\circ} \mathrm{C} /$} & $48 \% \mathrm{HzO}$ & 99 a & $100 \mathrm{a}$ & $6 \mathrm{~d}$ & $0 \mathrm{~d}$ & $0 \mathrm{c}$ \\
\hline & $43 \% \mathrm{H} 2 \mathrm{O}$ & $97 \mathrm{a}$ & $98 \mathrm{a}$ & $96 \mathrm{a}$ & $90 \mathrm{a}$ & $90 \mathrm{a}$ \\
\hline & $40 \% \mathrm{H} 2 \mathrm{O}$ & $82 \mathrm{~b}$ & $31 \mathrm{bc}$ & $35 \mathrm{c}$ & $38 \mathrm{~b}$ & $0 \mathrm{c}$ \\
\hline & $34 \% \mathrm{HzO}$ & $36 \mathrm{~d}$ & $21 \mathrm{~cd}$ & $5 d$ & $0 \mathrm{~d}$ & $0 \mathrm{c}$ \\
\hline & $30 \% \mathrm{H} 2 \mathrm{O}$ & $17 \mathrm{e}$ & $9 \mathrm{e}$ & $12 \mathrm{~d}$ & $1 \mathrm{~d}$ & $0 \mathrm{c}$ \\
\hline & $24 \% \mathrm{H} 2 \mathrm{O}$ & $0 \mathrm{f}$ & $0 \mathrm{f}$ & $0 \mathrm{e}$ & $0 \mathrm{~d}$ & $0 \mathrm{c}$ \\
\hline & $18 \% \mathrm{H} O$ & $0 \mathrm{f}$ & $0 \mathrm{f}$ & $0 \mathrm{e}$ & $0 \mathrm{~d}$ & $0 \mathrm{c}$ \\
\hline & $14 \% \mathrm{H} 2 \mathrm{O}$ & $0 \mathrm{f}$ & $0 \mathrm{f}$ & $0 \mathrm{e}$ & $0 \mathrm{~d}$ & $0 \mathrm{c}$ \\
\hline
\end{tabular}

1. Médias seguidas de mesma letra na coluna não diferem entre si, pelo teste de Tukey, ao nível de 5\% de probabilidade. 
Tabela 6. Emergência das plântulas de Myrciaria dubia: valores médios ${ }^{1}$ (\%) obtidos durante o armazenamento das sementes

\begin{tabular}{|c|c|c|c|c|c|c|}
\hline \multirow{2}{*}{\multicolumn{2}{|c|}{$\begin{array}{l}\text { Tratamentos (temperatura / } \\
\text { grau de umidade) }\end{array}$}} & \multicolumn{5}{|c|}{ Períodos de armazenamento (dias) } \\
\hline & & 35 & 70 & 140 & 210 & 280 \\
\hline \multicolumn{2}{|c|}{$30^{\circ} \mathrm{C} / 48 \% \mathrm{H} 2 \mathrm{O}$} & $25 \mathrm{~d}$ & $0 \mathrm{e}$ & $0 \mathrm{e}$ & $0 \mathrm{~d}$ & $0 \mathrm{c}$ \\
\hline & $43 \% \mathrm{H}_{2} \mathrm{O}$ & $94 a b$ & $17 \mathrm{bcd}$ & $0 \mathrm{e}$ & $0 \mathrm{~d}$ & $0 \mathrm{c}$ \\
\hline & $40 \% \mathrm{H} 2 \mathrm{O}$ & $0 \mathrm{f}$ & $0 \mathrm{e}$ & $0 \mathrm{e}$ & $0 \mathrm{~d}$ & $0 \mathrm{c}$ \\
\hline & $34 \% \mathrm{H} 2 \mathrm{O}$ & 2 ef & $0 \mathrm{e}$ & $0 \mathrm{e}$ & $0 \mathrm{~d}$ & $0 \mathrm{c}$ \\
\hline & $30 \% \mathrm{HzO}$ & $0 \mathrm{f}$ & $0 \mathrm{e}$ & $0 \mathrm{e}$ & $0 \mathrm{~d}$ & $0 \mathrm{c}$ \\
\hline & $24 \% \mathrm{H} 2 \mathrm{O}$ & $1 \mathrm{f}$ & $0 \mathrm{e}$ & $0 \mathrm{e}$ & $0 \mathrm{~d}$ & $0 \mathrm{c}$ \\
\hline & $18 \% \mathrm{H}$ & $0 \mathrm{f}$ & $0 \mathrm{e}$ & $0 \mathrm{e}$ & $0 \mathrm{~d}$ & $0 \mathrm{c}$ \\
\hline & $14 \% \mathrm{HzO}$ & $0 \mathrm{f}$ & $0 \mathrm{e}$ & $0 \mathrm{e}$ & $0 \mathrm{~d}$ & $0 \mathrm{c}$ \\
\hline \multirow[t]{8}{*}{$20^{\circ} \mathrm{C} /$} & $48 \% \mathrm{H} 2 \mathrm{O}$ & $99 \mathrm{a}$ & $98 \mathrm{a}$ & $0 \mathrm{e}$ & $0 \mathrm{~d}$ & $0 \mathrm{c}$ \\
\hline & $43 \% \mathrm{H}_{2} \mathrm{O}$ & $94 a b$ & $98 \mathrm{a}$ & $96 \mathrm{a}$ & $0 \mathrm{~d}$ & $0 \mathrm{c}$ \\
\hline & $40 \% \mathrm{HzO}$ & $58 \mathrm{c}$ & $37 \mathrm{~b}$ & $49 \mathrm{~b}$ & $0 \mathrm{~d}$ & $0 \mathrm{c}$ \\
\hline & $34 \% \mathrm{HzO}$ & $20 \mathrm{~d}$ & $26 \mathrm{bc}$ & $27 \mathrm{bc}$ & $19 \mathrm{c}$ & $3 \mathrm{~b}$ \\
\hline & $30 \% \mathrm{H} 2 \mathrm{O}$ & $22 \mathrm{~d}$ & $7 \mathrm{de}$ & $5 \mathrm{de}$ & $1 \mathrm{~d}$ & $0 \mathrm{c}$ \\
\hline & $24 \% \mathrm{H} 2 \mathrm{O}$ & 2 ef & $0 \mathrm{e}$ & $0 \mathrm{e}$ & $0 \mathrm{~d}$ & $0 \mathrm{c}$ \\
\hline & $18 \% \mathrm{H} 2 \mathrm{O}$ & $0 \mathrm{f}$ & $0 \mathrm{e}$ & $0 \mathrm{e}$ & $0 \mathrm{~d}$ & $0 \mathrm{c}$ \\
\hline & $14 \% \mathrm{HzO}$ & $0 \mathrm{f}$ & $0 \mathrm{e}$ & $0 \mathrm{e}$ & $0 \mathrm{~d}$ & $0 \mathrm{c}$ \\
\hline \multirow[t]{8}{*}{$10^{\circ} \mathrm{C} /$} & $48 \% \mathrm{HzO}$ & $94 a b$ & $97 \mathrm{a}$ & $9 \mathrm{~d}$ & $0 \mathrm{~d}$ & $0 \mathrm{c}$ \\
\hline & $43 \% \mathrm{H} 2 \mathrm{O}$ & $93 a b$ & $97 \mathrm{a}$ & $94 \mathrm{a}$ & $92 \mathrm{a}$ & $81 \mathrm{a}$ \\
\hline & $40 \% \mathrm{H} 2 \mathrm{O}$ & $80 \mathrm{bc}$ & $28 \mathrm{bc}$ & $36 \mathrm{~b}$ & $32 \mathrm{~b}$ & $0 \mathrm{c}$ \\
\hline & $34 \% \mathrm{HbO}$ & $29 \mathrm{~d}$ & $18 \mathrm{bc}$ & $3 \mathrm{de}$ & $0 \mathrm{~d}$ & $0 \mathrm{c}$ \\
\hline & $30 \% \mathrm{H} 2 \mathrm{O}$ & 16 de & $15 \mathrm{~cd}$ & $10 \mathrm{~cd}$ & $1 \mathrm{~d}$ & $0 \mathrm{c}$ \\
\hline & $24 \% \mathrm{H} 2 \mathrm{O}$ & $0 \mathrm{f}$ & $0 \mathrm{e}$ & $0 \mathrm{e}$ & $0 \mathrm{~d}$ & $0 \mathrm{c}$ \\
\hline & $18 \% \mathrm{H} O$ & $0 \mathrm{f}$ & $0 \mathrm{e}$ & $0 \mathrm{e}$ & $0 \mathrm{~d}$ & $0 \mathrm{c}$ \\
\hline & $14 \% \mathrm{H} 2 \mathrm{O}$ & $0 \mathrm{f}$ & $0 \mathrm{e}$ & $0 \mathrm{e}$ & $0 \mathrm{~d}$ & $0 \mathrm{c}$ \\
\hline
\end{tabular}

1. Médias seguidas de mesma letra na coluna não diferem entre si, pelo teste de Tukey, ao nível de 5\% de probabilidade. 
Tabela 7. Índice de velocidade de emergência das plântulas de Myrciaria dubia: valores médios ${ }^{1}$ obtidos durante o armazenamento das sementes

\begin{tabular}{|c|c|c|c|c|c|}
\hline \multirow{2}{*}{$\begin{array}{l}\text { Tratamentos (temperatura / } \\
\text { grau de umidade) }\end{array}$} & \multicolumn{5}{|c|}{ Períodos de armazenamento (dias) } \\
\hline & 35 & 70 & 140 & 210 & 280 \\
\hline $30^{\circ} \mathrm{C} / 48 \% \mathrm{H} 2 \mathrm{O}$ & 0,511 efg & $0,000 \mathrm{e}$ & $0,000 \mathrm{~d}$ & $0,000 \mathrm{~d}$ & $0,000 \mathrm{~b}$ \\
\hline $43 \% \mathrm{H} \mathrm{O}$ & $4,006 \mathrm{~b}$ & $0,436 \mathrm{de}$ & $0,000 \mathrm{~d}$ & $0,000 \mathrm{~d}$ & $0,000 \mathrm{~b}$ \\
\hline $40 \% \mathrm{H} \mathrm{O}$ & $0,000 \mathrm{~g}$ & $0,000 \mathrm{e}$ & $0,000 \mathrm{~d}$ & $0,000 \mathrm{~d}$ & $0,000 \mathrm{~b}$ \\
\hline $34 \% \mathrm{H} O$ & $0,040 \mathrm{fg}$ & $0,000 \mathrm{e}$ & $0,000 \mathrm{~d}$ & $0,000 \mathrm{~d}$ & $0,000 \mathrm{~b}$ \\
\hline $30 \% \mathrm{H} O$ & $0,000 \mathrm{~g}$ & $0,000 \mathrm{e}$ & $0,000 \mathrm{~d}$ & $0,000 \mathrm{~d}$ & $0,000 \mathrm{~b}$ \\
\hline $24 \% \mathrm{HbO}$ & $0,031 \mathrm{fg}$ & $0,000 \mathrm{e}$ & $0,000 \mathrm{~d}$ & $0,000 \mathrm{~d}$ & $0,000 \mathrm{~b}$ \\
\hline $18 \% \mathrm{H} O$ & $0,000 \mathrm{~g}$ & $0,000 \mathrm{e}$ & $0,000 \mathrm{~d}$ & $0,000 \mathrm{~d}$ & $0,000 \mathrm{~b}$ \\
\hline $14 \% \mathrm{HbO}$ & $0,000 \mathrm{~g}$ & $0,000 \mathrm{e}$ & $0,000 \mathrm{~d}$ & $0,000 \mathrm{~d}$ & $0,000 \mathrm{~b}$ \\
\hline $20^{\circ} \mathrm{C} / 48 \% \mathrm{HbO}$ & $4,683 \mathrm{a}$ & $5,180 \mathrm{a}$ & $0,000 \mathrm{~d}$ & $0,000 \mathrm{~d}$ & $0,000 \mathrm{~b}$ \\
\hline $43 \% \mathrm{H} O$ & 3,709 bc & $4,362 \mathrm{~b}$ & $4,460 \mathrm{a}$ & $0,000 \mathrm{~d}$ & $0,000 \mathrm{~b}$ \\
\hline $40 \% \mathrm{HbO}$ & $2,211 \mathrm{~d}$ & $1,412 \mathrm{c}$ & $2,082 \mathrm{~b}$ & $0,000 \mathrm{~d}$ & $0,000 \mathrm{~b}$ \\
\hline $34 \% \mathrm{H} O$ & $0,620 \mathrm{ef}$ & $0,973 \mathrm{~cd}$ & $0,994 \mathrm{c}$ & $0,901 \mathrm{c}$ & $0,054 \mathrm{~b}$ \\
\hline $30 \% \mathrm{H} \mathrm{O}$ & $0,692 \mathrm{e}$ & $0,202 \mathrm{e}$ & $0,115 \mathrm{~d}$ & $0,023 \mathrm{~d}$ & $0,000 \mathrm{~b}$ \\
\hline $24 \% \mathrm{HzO}$ & $0,068 \mathrm{fg}$ & $0,000 \mathrm{e}$ & $0,000 \mathrm{~d}$ & $0,000 \mathrm{~d}$ & $0,000 \mathrm{~b}$ \\
\hline $18 \% \mathrm{HbO}$ & $0,000 \mathrm{~g}$ & $0,000 \mathrm{e}$ & $0,000 \mathrm{~d}$ & $0,000 \mathrm{~d}$ & $0,000 \mathrm{~b}$ \\
\hline $14 \% \mathrm{H} O$ & $0,000 \mathrm{~g}$ & $0,000 \mathrm{e}$ & $0,000 \mathrm{~d}$ & $0,000 \mathrm{~d}$ & $0,000 \mathrm{~b}$ \\
\hline $10^{\circ} \mathrm{C} / 48 \% \mathrm{H} \mathrm{O}$ & $3,429 \mathrm{bc}$ & $4,335 \mathrm{~b}$ & $0,145 \mathrm{~d}$ & $0,000 \mathrm{~d}$ & $0,000 \mathrm{~b}$ \\
\hline $43 \% \mathrm{HbO}$ & $3,673 \mathrm{bc}$ & $4,247 \mathrm{~b}$ & $4,059 \mathrm{a}$ & $3,508 \mathrm{a}$ & $3,867 \mathrm{a}$ \\
\hline $40 \% \mathrm{HbO}$ & $3,289 \mathrm{c}$ & $0,498 \mathrm{de}$ & $1,294 \mathrm{c}$ & $1,363 \mathrm{~b}$ & $0,000 \mathrm{~b}$ \\
\hline $34 \% \mathrm{H} O$ & $0,898 \mathrm{e}$ & $0,474 \mathrm{de}$ & $0,054 \mathrm{~d}$ & $0,000 \mathrm{~d}$ & $0,000 \mathrm{~b}$ \\
\hline $30 \% \mathrm{HzO}$ & $0,530 \mathrm{efg}$ & $0,486 \mathrm{de}$ & $0,335 \mathrm{~d}$ & $0,023 \mathrm{~d}$ & $0,000 \mathrm{~b}$ \\
\hline $24 \% \mathrm{H} \mathrm{O}$ & $0,000 \mathrm{~g}$ & $0,000 \mathrm{e}$ & $0,000 \mathrm{~d}$ & $0,000 \mathrm{~d}$ & $0,000 \mathrm{~b}$ \\
\hline $18 \% \mathrm{HbO}$ & $0,000 \mathrm{~g}$ & $0,000 \mathrm{e}$ & $0,000 \mathrm{~d}$ & $0,000 \mathrm{~d}$ & $0,000 \mathrm{~b}$ \\
\hline $14 \% \mathrm{H} O$ & $0,000 \mathrm{~g}$ & $0,000 \mathrm{e}$ & $0,000 \mathrm{~d}$ & $0,000 \mathrm{~d}$ & $0,000 \mathrm{~b}$ \\
\hline
\end{tabular}

1. Médias seguidas de mesma letra na coluna não diferem entre si, pelo teste de Tukey, ao nível de 5\% de probabilidade. 
Tabela 8. Comprimento da plântula de Myrciaria dubia: valores médios ${ }^{1}$ ( $\mathrm{cm}$ ) obtidos durante o armazenamento das sementes

\begin{tabular}{|c|c|c|c|c|c|}
\hline \multirow{2}{*}{$\begin{array}{l}\text { Tratamentos (temperatura / } \\
\text { grau de umidade) }\end{array}$} & \multicolumn{5}{|c|}{ Períodos de armazenamento (dias) } \\
\hline & 35 & 70 & 140 & 210 & 280 \\
\hline $30^{\circ} \mathrm{C} / 48 \% \mathrm{H} 2 \mathrm{O}$ & $4,22 \mathrm{~d}$ & $0,00 \mathrm{e}$ & $0,00 \mathrm{e}$ & $0,00 \mathrm{~d}$ & $0,00 \mathrm{~b}$ \\
\hline $43 \% \mathrm{HzO}$ & 24,69 a & $4,06 \mathrm{~cd}$ & $0,00 \mathrm{e}$ & $0,00 \mathrm{~d}$ & $0,00 \mathrm{~b}$ \\
\hline $40 \% \mathrm{HzO}$ & $0,00 \mathrm{f}$ & $0,00 \mathrm{e}$ & $0,00 \mathrm{e}$ & $0,00 \mathrm{~d}$ & $0,00 \mathrm{~b}$ \\
\hline $34 \% \mathrm{H} O$ & 0,26 ef & $0,00 \mathrm{e}$ & $0,00 \mathrm{e}$ & $0,00 \mathrm{~d}$ & $0,00 \mathrm{~b}$ \\
\hline $30 \% \mathrm{H} O$ & $0,00 \mathrm{f}$ & $0,00 \mathrm{e}$ & $0,00 \mathrm{e}$ & $0,00 \mathrm{~d}$ & $0,00 \mathrm{~b}$ \\
\hline $24 \% \mathrm{HzO}$ & 0,14 ef & $0,00 \mathrm{e}$ & $0,00 \mathrm{e}$ & $0,00 \mathrm{~d}$ & $0,00 \mathrm{~b}$ \\
\hline $18 \% \mathrm{HzO}$ & $0,00 \mathrm{f}$ & $0,00 \mathrm{e}$ & $0,00 \mathrm{e}$ & $0,00 \mathrm{~d}$ & $0,00 \mathrm{~b}$ \\
\hline $14 \% \mathrm{HzO}$ & $0,00 \mathrm{f}$ & $0,00 \mathrm{e}$ & $0,00 \mathrm{e}$ & $0,00 \mathrm{~d}$ & $0,00 \mathrm{~b}$ \\
\hline $20^{\circ} \mathrm{C} / 48 \% \mathrm{HzO}$ & $27,74 \mathrm{a}$ & $27,47 \mathrm{a}$ & $0,00 \mathrm{e}$ & $0,00 \mathrm{~d}$ & $0,00 \mathrm{~b}$ \\
\hline $43 \% \mathrm{H} O$ & $25,51 \mathrm{a}$ & $26,22 \mathrm{a}$ & $27,89 \mathrm{a}$ & $0,00 \mathrm{~d}$ & $0,00 \mathrm{~b}$ \\
\hline $40 \% \mathrm{HzO}$ & $13,48 \mathrm{c}$ & $8,25 \mathrm{~b}$ & $11,37 \mathrm{~b}$ & $0,00 \mathrm{~d}$ & $0,00 \mathrm{~b}$ \\
\hline $34 \% \mathrm{H} \mathrm{O}$ & $3,23 \mathrm{def}$ & $5,86 \mathrm{bc}$ & $5,31 \mathrm{~cd}$ & $4,17 \mathrm{c}$ & $0,49 b$ \\
\hline $30 \% \mathrm{H} O$ & $3,75 \mathrm{de}$ & $1,43 \mathrm{de}$ & $0,91 \mathrm{e}$ & $0,23 \mathrm{~d}$ & $0,00 \mathrm{~b}$ \\
\hline $24 \% \mathrm{H} 2 \mathrm{O}$ & 0,29 ef & $0,00 \mathrm{e}$ & $0,00 \mathrm{e}$ & $0,00 \mathrm{~d}$ & $0,00 \mathrm{~b}$ \\
\hline $18 \% \mathrm{HzO}$ & $0,00 \mathrm{f}$ & $0,00 \mathrm{e}$ & $0,00 \mathrm{e}$ & $0,00 \mathrm{~d}$ & $0,00 \mathrm{~b}$ \\
\hline $14 \% \mathrm{HzO}$ & $0,00 \mathrm{f}$ & $0,00 \mathrm{e}$ & $0,00 \mathrm{e}$ & $0,00 \mathrm{~d}$ & $0,00 \mathrm{~b}$ \\
\hline $10^{\circ} \mathrm{C} / 48 \% \mathrm{HzO}$ & $25,55 \mathrm{a}$ & $26,84 \mathrm{a}$ & $1,68 \mathrm{e}$ & $0,00 \mathrm{~d}$ & $0,00 \mathrm{~b}$ \\
\hline $43 \% \mathrm{HbO}$ & $25,35 \mathrm{a}$ & $29,28 \mathrm{a}$ & $26,07 \mathrm{a}$ & $25,18 \mathrm{a}$ & $20,34 \mathrm{a}$ \\
\hline $40 \% \mathrm{HzO}$ & $19,41 b$ & $4,93 \mathrm{c}$ & $7,91 \mathrm{bc}$ & $7,09 \mathrm{~b}$ & $0,00 \mathrm{~b}$ \\
\hline $34 \% \mathrm{HzO}$ & $4,52 \mathrm{~d}$ & $3,48 \mathrm{~cd}$ & $0,32 \mathrm{e}$ & $0,00 \mathrm{~d}$ & $0,00 \mathrm{~b}$ \\
\hline $30 \% \mathrm{H} 2 \mathrm{O}$ & 2,77 def & $3,55 \mathrm{~cd}$ & $2,13 \mathrm{de}$ & $0,13 \mathrm{~d}$ & $0,00 \mathrm{~b}$ \\
\hline $24 \% \mathrm{H} O$ & $0,00 \mathrm{f}$ & $0,00 \mathrm{e}$ & $0,00 \mathrm{e}$ & $0,00 \mathrm{~d}$ & $0,00 \mathrm{~b}$ \\
\hline $18 \% \mathrm{HbO}$ & $0,00 \mathrm{f}$ & $0,00 \mathrm{e}$ & $0,00 \mathrm{e}$ & $0,00 \mathrm{~d}$ & $0,00 \mathrm{~b}$ \\
\hline $14 \% \mathrm{H} 2 \mathrm{O}$ & $0,00 \mathrm{f}$ & $0,00 \mathrm{e}$ & $0,00 \mathrm{e}$ & $0,00 \mathrm{~d}$ & $0,00 \mathrm{~b}$ \\
\hline
\end{tabular}

1. Médias seguidas de mesma letra na coluna não diferem entre si, pelo teste de Tukey, ao nível de $5 \%$ de probabilidade. 
Tabela 9. Diâmetro do colo da plântula de Myrciaria dubia: valores médios ${ }^{1}$ (mm) obtidos durante o armazenamento das sementes

\begin{tabular}{|c|c|c|c|c|c|}
\hline \multirow{2}{*}{$\begin{array}{l}\text { Tratamentos (temperatura / } \\
\text { grau de umidade) }\end{array}$} & \multicolumn{5}{|c|}{ Períodos de armazenamento (dias) } \\
\hline & 35 & 70 & 140 & 210 & 280 \\
\hline $30^{\circ} \mathrm{C} / 48 \% \mathrm{HzO}$ & $0,26 \mathrm{ef}$ & $0,00 \mathrm{e}$ & $0,00 \mathrm{e}$ & $0,00 \mathrm{~d}$ & $0,00 \mathrm{~b}$ \\
\hline $43 \% \mathrm{H} O$ & $1,68 \mathrm{ab}$ & $0,22 \mathrm{~cd}$ & $0,00 \mathrm{e}$ & $0,00 \mathrm{~d}$ & $0,00 \mathrm{~b}$ \\
\hline $40 \% \mathrm{H} 2 \mathrm{O}$ & $0,00 \mathrm{f}$ & $0,00 \mathrm{e}$ & $0,00 \mathrm{e}$ & $0,00 \mathrm{~d}$ & $0,00 \mathrm{~b}$ \\
\hline $34 \% \mathrm{H} 2 \mathrm{O}$ & $0,02 \mathrm{f}$ & $0,00 \mathrm{e}$ & $0,00 \mathrm{e}$ & $0,00 \mathrm{~d}$ & $0,00 \mathrm{~b}$ \\
\hline $30 \% \mathrm{H} O$ & $0,00 \mathrm{f}$ & $0,00 \mathrm{e}$ & $0,00 \mathrm{e}$ & $0,00 \mathrm{~d}$ & $0,00 \mathrm{~b}$ \\
\hline $24 \% \mathrm{H} O$ & $0,01 \mathrm{f}$ & $0,00 \mathrm{e}$ & $0,00 \mathrm{e}$ & $0,00 \mathrm{~d}$ & $0,00 \mathrm{~b}$ \\
\hline $18 \% \mathrm{H} 2 \mathrm{O}$ & $0,00 \mathrm{f}$ & $0,00 \mathrm{e}$ & $0,00 \mathrm{e}$ & $0,00 \mathrm{~d}$ & $0,00 \mathrm{~b}$ \\
\hline $14 \% \mathrm{HzO}$ & $0,00 \mathrm{f}$ & $0,00 \mathrm{e}$ & $0,00 \mathrm{e}$ & $0,00 \mathrm{~d}$ & $0,00 \mathrm{~b}$ \\
\hline $20^{\circ} \mathrm{C} / 48 \% \mathrm{HzO}$ & $1,91 \mathrm{a}$ & $1,70 \mathrm{a}$ & $0,00 \mathrm{e}$ & $0,00 \mathrm{~d}$ & $0,00 \mathrm{~b}$ \\
\hline $43 \% \mathrm{H} 2 \mathrm{O}$ & $1,70 \mathrm{ab}$ & $1,67 \mathrm{a}$ & $1,46 \mathrm{a}$ & $0,00 \mathrm{~d}$ & $0,00 \mathrm{~b}$ \\
\hline $40 \% \mathrm{H} O$ & $0,94 \mathrm{~d}$ & $0,47 \mathrm{~b}$ & $0,62 \mathrm{~b}$ & $0,00 \mathrm{~d}$ & $0,00 \mathrm{~b}$ \\
\hline $34 \% \mathrm{H} O$ & $0,20 \mathrm{ef}$ & $0,38 \mathrm{bc}$ & $0,27 \mathrm{~cd}$ & $0,25 \mathrm{c}$ & $0,03 \mathrm{~b}$ \\
\hline $30 \% \mathrm{H} 2 \mathrm{O}$ & 0,26 ef & $0,08 \mathrm{de}$ & $0,05 \mathrm{e}$ & $0,01 \mathrm{~d}$ & $0,00 \mathrm{~b}$ \\
\hline $24 \% \mathrm{H} 2 \mathrm{O}$ & $0,02 \mathrm{f}$ & $0,00 \mathrm{e}$ & $0,00 \mathrm{e}$ & $0,00 \mathrm{~d}$ & $0,00 \mathrm{~b}$ \\
\hline $18 \% \mathrm{HzO}$ & $0,00 \mathrm{f}$ & $0,00 \mathrm{e}$ & $0,00 \mathrm{e}$ & $0,00 \mathrm{~d}$ & $0,00 \mathrm{~b}$ \\
\hline $14 \% \mathrm{H} 2 \mathrm{O}$ & $0,00 \mathrm{f}$ & $0,00 \mathrm{e}$ & $0,00 \mathrm{e}$ & $0,00 \mathrm{~d}$ & $0,00 \mathrm{~b}$ \\
\hline $10^{\circ} \mathrm{C} / 48 \% \mathrm{HzO}$ & $1,56 \mathrm{bc}$ & $1,66 \mathrm{a}$ & $0,09 \mathrm{de}$ & $0,00 \mathrm{~d}$ & $0,00 \mathrm{~b}$ \\
\hline $43 \% \mathrm{HzO}$ & $1,62 \mathrm{~b}$ & $1,77 \mathrm{a}$ & $1,40 \mathrm{a}$ & $1,34 \mathrm{a}$ & $1,27 \mathrm{a}$ \\
\hline $40 \% \mathrm{H} O$ & $1,30 \mathrm{c}$ & $0,36 \mathrm{bc}$ & $0,39 \mathrm{c}$ & $0,42 \mathrm{~b}$ & $0,00 \mathrm{~b}$ \\
\hline $34 \% \mathrm{HzO}$ & $0,31 \mathrm{e}$ & $0,23 \mathrm{~cd}$ & $0,02 \mathrm{e}$ & $0,00 \mathrm{~d}$ & $0,00 \mathrm{~b}$ \\
\hline $30 \% \mathrm{H} 2 \mathrm{O}$ & 0,18 ef & $0,21 \mathrm{~cd}$ & $0,10 \mathrm{de}$ & $0,01 \mathrm{~d}$ & $0,00 \mathrm{~b}$ \\
\hline $24 \% \mathrm{H} 2 \mathrm{O}$ & $0,00 \mathrm{f}$ & $0,00 \mathrm{e}$ & $0,00 \mathrm{e}$ & $0,00 \mathrm{~d}$ & $0,00 \mathrm{~b}$ \\
\hline $18 \% \mathrm{H} O$ & $0,00 \mathrm{f}$ & $0,00 \mathrm{e}$ & $0,00 \mathrm{e}$ & $0,00 \mathrm{~d}$ & $0,00 \mathrm{~b}$ \\
\hline $14 \% \mathrm{H} 2 \mathrm{O}$ & $0,00 \mathrm{f}$ & $0,00 \mathrm{e}$ & $0,00 \mathrm{e}$ & $0,00 \mathrm{~d}$ & $0,00 \mathrm{~b}$ \\
\hline
\end{tabular}

1. Médias seguidas de mesma letra na coluna não diferem entre si, pelo teste de Tukey, ao nível de 5\% de probabilidade. 
Tabela 10. Número de folhas da plântula de Myrciaria dubia: valores médios ${ }^{1}$ obtidos durante o armazenamento das sementes

\begin{tabular}{|c|c|c|c|c|c|}
\hline \multirow{2}{*}{$\begin{array}{l}\text { Tratamentos (temperatura / } \\
\text { grau de umidade) }\end{array}$} & \multicolumn{5}{|c|}{ Períodos de armazenamento (dias) } \\
\hline & 35 & 70 & 140 & 210 & 280 \\
\hline $30^{\circ} \mathrm{C} / 48 \% \mathrm{HzO}$ & $2,48 \mathrm{c}$ & $0,00 \mathrm{e}$ & $0,00 \mathrm{~g}$ & $0,00 \mathrm{~d}$ & $0,00 \mathrm{c}$ \\
\hline $43 \% \mathrm{H} 2 \mathrm{O}$ & $13,40 \mathrm{a}$ & $2,22 \mathrm{bc}$ & $0,00 \mathrm{~g}$ & $0,00 \mathrm{~d}$ & $0,00 \mathrm{c}$ \\
\hline $40 \% \mathrm{H} 2 \mathrm{O}$ & $0,00 \mathrm{e}$ & $0,00 \mathrm{e}$ & $0,00 \mathrm{~g}$ & $0,00 \mathrm{~d}$ & $0,00 \mathrm{c}$ \\
\hline $34 \% \mathrm{H} 2 \mathrm{O}$ & $0,13 \mathrm{de}$ & $0,00 \mathrm{e}$ & $0,00 \mathrm{~g}$ & $0,00 \mathrm{~d}$ & $0,00 \mathrm{c}$ \\
\hline $30 \% \mathrm{H} O$ & $0,00 \mathrm{e}$ & $0,00 \mathrm{e}$ & $0,00 \mathrm{~g}$ & $0,00 \mathrm{~d}$ & $0,00 \mathrm{c}$ \\
\hline $24 \% \mathrm{H} O$ & $0,08 \mathrm{e}$ & $0,00 \mathrm{e}$ & $0,00 \mathrm{~g}$ & $0,00 \mathrm{~d}$ & $0,00 \mathrm{c}$ \\
\hline $18 \% \mathrm{HzO}$ & $0,00 \mathrm{e}$ & $0,00 \mathrm{e}$ & $0,00 \mathrm{~g}$ & $0,00 \mathrm{~d}$ & $0,00 \mathrm{c}$ \\
\hline $14 \% \mathrm{HzO}$ & $0,00 \mathrm{e}$ & $0,00 \mathrm{e}$ & $0,00 \mathrm{~g}$ & $0,00 \mathrm{~d}$ & $0,00 \mathrm{c}$ \\
\hline $20^{\circ} \mathrm{C} / 48 \% \mathrm{H} \mathrm{O}$ & $15,57 \mathrm{a}$ & $15,48 \mathrm{a}$ & $0,00 \mathrm{~g}$ & $0,00 \mathrm{~d}$ & $0,00 \mathrm{c}$ \\
\hline $43 \% \mathrm{H} 2 \mathrm{O}$ & $14,11 \mathrm{a}$ & $13,88 \mathrm{a}$ & $14,34 \mathrm{a}$ & $0,00 \mathrm{~d}$ & $0,00 \mathrm{c}$ \\
\hline $40 \% \mathrm{HzO}$ & $7,82 \mathrm{~b}$ & $3,92 \mathrm{~b}$ & $6,39 \mathrm{~b}$ & $0,00 \mathrm{~d}$ & $0,00 \mathrm{c}$ \\
\hline $34 \% \mathrm{HzO}$ & $1,58 \mathrm{c}$ & $3,11 \mathrm{bc}$ & $2,51 \mathrm{~cd}$ & $2,37 \mathrm{c}$ & $0,27 \mathrm{~b}$ \\
\hline $30 \% \mathrm{HzO}$ & $2,00 \mathrm{c}$ & $0,66 \mathrm{~d}$ & 0,47 efg & $0,14 \mathrm{~d}$ & $0,00 \mathrm{c}$ \\
\hline $24 \% \mathrm{H} 2 \mathrm{O}$ & $0,18 \mathrm{de}$ & $0,00 \mathrm{e}$ & $0,00 \mathrm{~g}$ & $0,00 \mathrm{~d}$ & $0,00 \mathrm{c}$ \\
\hline $18 \% \mathrm{H} 2 \mathrm{O}$ & $0,00 \mathrm{e}$ & $0,00 \mathrm{e}$ & $0,00 \mathrm{~g}$ & $0,00 \mathrm{~d}$ & $0,00 \mathrm{c}$ \\
\hline $14 \% \mathrm{HzO}$ & $0,00 \mathrm{e}$ & $0,00 \mathrm{e}$ & $0,00 \mathrm{~g}$ & $0,00 \mathrm{~d}$ & $0,00 \mathrm{c}$ \\
\hline $10^{\circ} \mathrm{C} / 48 \% \mathrm{HzO}$ & $13,75 \mathrm{a}$ & $13,88 \mathrm{a}$ & 0,86 ef & $0,00 \mathrm{~d}$ & $0,00 \mathrm{c}$ \\
\hline $43 \% \mathrm{HzO}$ & $13,46 \mathrm{a}$ & $15,23 \mathrm{a}$ & $13,56 \mathrm{a}$ & $13,24 \mathrm{a}$ & $11,47 \mathrm{a}$ \\
\hline $40 \% \mathrm{H} 2 \mathrm{O}$ & $10,35 \mathrm{ab}$ & $2,85 \mathrm{bc}$ & $3,99 \mathrm{bc}$ & $3,48 \mathrm{~b}$ & $0,00 \mathrm{c}$ \\
\hline $34 \% \mathrm{HzO}$ & $2,70 \mathrm{c}$ & $1,96 \mathrm{c}$ & $0,12 \mathrm{fg}$ & $0,00 \mathrm{~d}$ & $0,00 \mathrm{c}$ \\
\hline $30 \% \mathrm{H} 2 \mathrm{O}$ & $1,44 \mathrm{~cd}$ & $1,82 \mathrm{c}$ & $1,25 \mathrm{de}$ & $0,09 \mathrm{~d}$ & $0,00 \mathrm{c}$ \\
\hline $24 \% \mathrm{H} 2 \mathrm{O}$ & $0,00 \mathrm{e}$ & $0,00 \mathrm{e}$ & $0,00 \mathrm{~g}$ & $0,00 \mathrm{~d}$ & $0,00 \mathrm{c}$ \\
\hline $18 \% \mathrm{H} 2 \mathrm{O}$ & $0,00 \mathrm{e}$ & $0,00 \mathrm{e}$ & $0,00 \mathrm{~g}$ & $0,00 \mathrm{~d}$ & $0,00 \mathrm{c}$ \\
\hline $14 \% \mathrm{HzO}$ & $0,00 \mathrm{e}$ & $0,00 \mathrm{e}$ & $0,00 \mathrm{~g}$ & $0,00 \mathrm{~d}$ & $0,00 \mathrm{c}$ \\
\hline
\end{tabular}

1. Médias seguidas de mesma letra na coluna não diferem entre si, pelo teste de Tukey, ao nível de 5\% de probabilidade. 
Tabela 11. Massa da matéria seca da plântula de Myrciaria dubia: valores médios ${ }^{1}$ (g) obtidos durante o armazenamento das sementes

\begin{tabular}{|c|c|c|c|c|c|}
\hline \multirow{2}{*}{$\begin{array}{l}\text { Tratamentos (temperatura / } \\
\text { grau de umidade) }\end{array}$} & \multicolumn{5}{|c|}{ Períodos de armazenamento (dias) } \\
\hline & 35 & 70 & 140 & 210 & 280 \\
\hline $30^{\circ} \mathrm{C} / 48 \% \mathrm{HzO}$ & $0,026 \mathrm{~d}$ & $0,000 \mathrm{f}$ & $0,000 \mathrm{e}$ & $0,000 \mathrm{~d}$ & $0,000 \mathrm{~b}$ \\
\hline $43 \% \mathrm{H} 2 \mathrm{O}$ & $0,339 \mathrm{ab}$ & 0,034 def & $0,000 \mathrm{e}$ & $0,000 \mathrm{~d}$ & $0,000 \mathrm{~b}$ \\
\hline $40 \% \mathrm{H} 2 \mathrm{O}$ & $0,000 \mathrm{~d}$ & $0,000 \mathrm{f}$ & $0,000 \mathrm{e}$ & $0,000 \mathrm{~d}$ & $0,000 \mathrm{~b}$ \\
\hline $34 \% \mathrm{H} 2 \mathrm{O}$ & $0,001 \mathrm{~d}$ & $0,000 \mathrm{f}$ & $0,000 \mathrm{e}$ & $0,000 \mathrm{~d}$ & $0,000 \mathrm{~b}$ \\
\hline $30 \% \mathrm{H} O$ & $0,000 \mathrm{~d}$ & $0,000 \mathrm{f}$ & $0,000 \mathrm{e}$ & $0,000 \mathrm{~d}$ & $0,000 \mathrm{~b}$ \\
\hline $24 \% \mathrm{H} O$ & $0,000 \mathrm{~d}$ & $0,000 \mathrm{f}$ & $0,000 \mathrm{e}$ & $0,000 \mathrm{~d}$ & $0,000 \mathrm{~b}$ \\
\hline $18 \% \mathrm{HzO}$ & $0,000 \mathrm{~d}$ & $0,000 \mathrm{f}$ & $0,000 \mathrm{e}$ & $0,000 \mathrm{~d}$ & $0,000 \mathrm{~b}$ \\
\hline $14 \% \mathrm{HzO}$ & $0,000 \mathrm{~d}$ & $0,000 \mathrm{f}$ & $0,000 \mathrm{e}$ & $0,000 \mathrm{~d}$ & $0,000 \mathrm{~b}$ \\
\hline $20^{\circ} \mathrm{C} / 48 \% \mathrm{HzO}$ & $0,407 \mathrm{a}$ & $0,431 \mathrm{a}$ & $0,000 \mathrm{e}$ & $0,000 \mathrm{~d}$ & $0,000 \mathrm{~b}$ \\
\hline $43 \% \mathrm{H} 2 \mathrm{O}$ & $0,337 \mathrm{ab}$ & $0,356 \mathrm{c}$ & $0,333 \mathrm{a}$ & $0,000 \mathrm{~d}$ & $0,000 \mathrm{~b}$ \\
\hline $40 \% \mathrm{HzO}$ & $0,144 \mathrm{c}$ & $0,076 \mathrm{~d}$ & $0,109 \mathrm{c}$ & $0,000 \mathrm{~d}$ & $0,000 \mathrm{~b}$ \\
\hline $34 \% \mathrm{HzO}$ & $0,024 \mathrm{~d}$ & $0,067 \mathrm{de}$ & 0,034 de & $0,035 \mathrm{c}$ & $0,001 \mathrm{~b}$ \\
\hline $30 \% \mathrm{H} 2 \mathrm{O}$ & $0,029 \mathrm{~d}$ & 0,010 ef & $0,003 \mathrm{e}$ & $0,001 \mathrm{~d}$ & $0,000 \mathrm{~b}$ \\
\hline $24 \% \mathrm{H} 2 \mathrm{O}$ & $0,001 \mathrm{~d}$ & $0,000 \mathrm{f}$ & $0,000 \mathrm{e}$ & $0,000 \mathrm{~d}$ & $0,000 \mathrm{~b}$ \\
\hline $18 \% \mathrm{H} 2 \mathrm{O}$ & $0,000 \mathrm{~d}$ & $0,000 \mathrm{f}$ & $0,000 \mathrm{e}$ & $0,000 \mathrm{~d}$ & $0,000 \mathrm{~b}$ \\
\hline $14 \% \mathrm{HzO}$ & $0,000 \mathrm{~d}$ & $0,000 \mathrm{f}$ & $0,000 \mathrm{e}$ & $0,000 \mathrm{~d}$ & $0,000 \mathrm{~b}$ \\
\hline $10^{\circ} \mathrm{C} / 48 \% \mathrm{HzO}$ & $0,305 \mathrm{~b}$ & $0,364 \mathrm{bc}$ & $0,006 \mathrm{e}$ & $0,000 \mathrm{~d}$ & $0,000 \mathrm{~b}$ \\
\hline $43 \% \mathrm{HzO}$ & $0,328 \mathrm{~b}$ & $0,419 \mathrm{ab}$ & $0,282 \mathrm{~b}$ & $0,257 \mathrm{a}$ & $0,231 \mathrm{a}$ \\
\hline $40 \% \mathrm{H} 2 \mathrm{O}$ & $0,214 \mathrm{c}$ & 0,032 def & $0,054 \mathrm{~d}$ & $0,057 \mathrm{~b}$ & $0,000 \mathrm{~b}$ \\
\hline $34 \% \mathrm{HzO}$ & $0,036 \mathrm{~d}$ & 0,029 def & $0,000 \mathrm{e}$ & $0,000 \mathrm{~d}$ & $0,000 \mathrm{~b}$ \\
\hline $30 \% \mathrm{H} \mathrm{O}$ & $0,019 \mathrm{~d}$ & $0,036 \mathrm{def}$ & $0,012 \mathrm{e}$ & $0,000 \mathrm{~d}$ & $0,000 \mathrm{~b}$ \\
\hline $24 \% \mathrm{H}$ & $0,000 \mathrm{~d}$ & $0,000 \mathrm{f}$ & $0,000 \mathrm{e}$ & $0,000 \mathrm{~d}$ & $0,000 \mathrm{~b}$ \\
\hline $18 \% \mathrm{H} O$ & $0,000 \mathrm{~d}$ & $0,000 \mathrm{f}$ & $0,000 \mathrm{e}$ & $0,000 \mathrm{~d}$ & $0,000 \mathrm{~b}$ \\
\hline $14 \% \mathrm{HzO}$ & $0,000 \mathrm{~d}$ & $0,000 \mathrm{f}$ & $0,000 \mathrm{e}$ & $0,000 \mathrm{~d}$ & $0,000 \mathrm{~b}$ \\
\hline
\end{tabular}

1. Médias seguidas de mesma letra na coluna não diferem entre si, pelo teste de Tukey, ao nível de 5\% de probabilidade. 
Tabela 12. Incidência de Fusarium sp. nas sementes de Myrciaria dubia: valores médios $^{1}(\%)$ obtidos durante o armazenamento

\begin{tabular}{|c|c|c|c|c|c|c|}
\hline \multirow{2}{*}{\multicolumn{2}{|c|}{$\begin{array}{l}\text { Tratamentos (temperatura / } \\
\text { grau de umidade) }\end{array}$}} & \multicolumn{5}{|c|}{ Períodos de armazenamento (dias) } \\
\hline & & 35 & 70 & 140 & 210 & 280 \\
\hline \multirow[t]{8}{*}{$30^{\circ} \mathrm{C} /$} & $48 \% \mathrm{H} 2 \mathrm{O}$ & $94 \mathrm{ab}$ & $100 \mathrm{a}$ & $52 \mathrm{c}$ & $12 \mathrm{de}$ & $100 \mathrm{a}$ \\
\hline & $43 \% \mathrm{HzO}$ & 80 abcd & $100 \mathrm{a}$ & $98 \mathrm{a}$ & $100 \mathrm{a}$ & $84 \mathrm{~b}$ \\
\hline & $40 \% \mathrm{HzO}$ & $94 \mathrm{ab}$ & $100 \mathrm{a}$ & $100 \mathrm{a}$ & $100 \mathrm{a}$ & $100 \mathrm{a}$ \\
\hline & $34 \% \mathrm{HzO}$ & $100 \mathrm{a}$ & $100 \mathrm{a}$ & $100 \mathrm{a}$ & $100 \mathrm{a}$ & $100 \mathrm{a}$ \\
\hline & $30 \% \mathrm{H} O$ & $100 \mathrm{a}$ & $100 \mathrm{a}$ & $100 \mathrm{a}$ & $100 \mathrm{a}$ & $72 \mathrm{~b}$ \\
\hline & $24 \% \mathrm{H}$ & $0 \mathrm{~g}$ & $84 \mathrm{abc}$ & $46 c$ & $0 \mathrm{e}$ & $0 \mathrm{e}$ \\
\hline & $18 \% \mathrm{HbO}$ & $0 \mathrm{~g}$ & $0 \mathrm{~h}$ & $0 \mathrm{~d}$ & $0 \mathrm{e}$ & $34 \mathrm{~cd}$ \\
\hline & $14 \% \mathrm{H}$ & $12 \mathrm{efg}$ & $12 \mathrm{fgh}$ & $0 \mathrm{~d}$ & $0 \mathrm{e}$ & $0 \mathrm{e}$ \\
\hline \multirow[t]{8}{*}{$20^{\circ} \mathrm{C} /$} & $48 \% \mathrm{H} 2 \mathrm{O}$ & 36 defg & 30 defgh & $34 c$ & $2 \mathrm{e}$ & $0 \mathrm{e}$ \\
\hline & $43 \% \mathrm{HbO}$ & 76 abcd & $92 a b$ & $100 \mathrm{a}$ & $50 \mathrm{c}$ & $20 \mathrm{~d}$ \\
\hline & $40 \% \mathrm{HzO}$ & $86 \mathrm{abc}$ & 60 cde & $100 \mathrm{a}$ & $100 \mathrm{a}$ & $100 \mathrm{a}$ \\
\hline & $34 \% \mathrm{H} 2 \mathrm{O}$ & $96 \mathrm{ab}$ & $100 \mathrm{a}$ & $100 \mathrm{a}$ & $100 \mathrm{a}$ & $100 \mathrm{a}$ \\
\hline & $30 \% \mathrm{HbO}$ & 80 abcd & $100 \mathrm{a}$ & $100 \mathrm{a}$ & $100 \mathrm{a}$ & $100 \mathrm{a}$ \\
\hline & $24 \% \mathrm{H2O}$ & $0 \mathrm{~g}$ & 8 gh & $0 \mathrm{~d}$ & $0 \mathrm{e}$ & $0 \mathrm{e}$ \\
\hline & $18 \% \mathrm{H}$ & $4 \mathrm{fg}$ & $0 \mathrm{~h}$ & $0 \mathrm{~d}$ & $0 \mathrm{e}$ & $0 \mathrm{e}$ \\
\hline & $14 \% \mathrm{HbO}$ & 42 defg & 24 defgh & $12 \mathrm{~d}$ & $0 \mathrm{e}$ & $0 \mathrm{e}$ \\
\hline \multirow[t]{8}{*}{$10^{\circ} \mathrm{C} /$} & $48 \% \mathrm{HbO}$ & 70 abcd & $2 \mathrm{~h}$ & $44 \mathrm{c}$ & $84 \mathrm{~b}$ & $38 \mathrm{c}$ \\
\hline & $43 \% \mathrm{H} 2 \mathrm{O}$ & $46 \mathrm{cdef}$ & 16 efgh & $84 \mathrm{~b}$ & $94 \mathrm{ab}$ & $100 \mathrm{a}$ \\
\hline & $40 \% \mathrm{HzO}$ & 48 cdef & 54 cdef & $100 \mathrm{a}$ & $20 \mathrm{~cd}$ & $96 \mathrm{a}$ \\
\hline & $34 \% \mathrm{H} 2 \mathrm{O}$ & 60 bcde & $64 \mathrm{bcd}$ & $98 \mathrm{a}$ & $96 \mathrm{ab}$ & $100 \mathrm{a}$ \\
\hline & $30 \% \mathrm{H} 2 \mathrm{O}$ & $16 \mathrm{efg}$ & $100 \mathrm{a}$ & $100 \mathrm{a}$ & $100 \mathrm{a}$ & $100 \mathrm{a}$ \\
\hline & $24 \% \mathrm{H} O$ & $0 \mathrm{~g}$ & $0 \mathrm{~h}$ & $0 \mathrm{~d}$ & $0 \mathrm{e}$ & $0 \mathrm{e}$ \\
\hline & $18 \% \mathrm{HzO}$ & 42 defg & 42 cdefg & $0 \mathrm{~d}$ & $0 \mathrm{e}$ & $0 \mathrm{e}$ \\
\hline & $14 \% \mathrm{H} 2 \mathrm{O}$ & 60 bcde & 30 defgh & $50 \mathrm{c}$ & $0 \mathrm{e}$ & $0 \mathrm{e}$ \\
\hline
\end{tabular}

1. Médias seguidas de mesma letra na coluna não diferem entre si, pelo teste de Tukey, ao nível de 5\% de probabilidade. 
Tabela 13. Incidência de Penicillium sp. nas sementes de Myrciaria dubia: valores médios $^{1}(\%)$ obtidos durante o armazenamento

\begin{tabular}{|c|c|c|c|c|c|c|}
\hline \multirow{2}{*}{\multicolumn{2}{|c|}{$\begin{array}{l}\text { Tratamentos (temperatura / } \\
\text { grau de umidade) }\end{array}$}} & \multicolumn{5}{|c|}{ Períodos de armazenamento (dias) } \\
\hline & & 35 & 70 & 140 & 210 & 280 \\
\hline \multirow[t]{8}{*}{$30^{\circ} \mathrm{C} /$} & $48 \% \mathrm{HbO}$ & $4 \mathrm{de}$ & $6 \mathrm{e}$ & $38 \mathrm{de}$ & $20 \mathrm{~d}$ & $0 \mathrm{e}$ \\
\hline & $43 \% \mathrm{H} O$ & $0 \mathrm{e}$ & $0 \mathrm{e}$ & $0 \mathrm{f}$ & $0 \mathrm{e}$ & $0 \mathrm{e}$ \\
\hline & $40 \% \mathrm{H} O$ & $4 \mathrm{de}$ & $0 \mathrm{e}$ & $0 \mathrm{f}$ & $0 \mathrm{e}$ & $0 \mathrm{e}$ \\
\hline & $34 \% \mathrm{HzO}$ & $0 \mathrm{e}$ & $0 \mathrm{e}$ & $0 \mathrm{f}$ & $0 \mathrm{e}$ & $0 \mathrm{e}$ \\
\hline & $30 \% \mathrm{HbO}$ & $0 \mathrm{e}$ & $0 \mathrm{e}$ & $0 \mathrm{f}$ & $14 \mathrm{~d}$ & $10 \mathrm{de}$ \\
\hline & $24 \% \mathrm{H} O$ & $86 \mathrm{ab}$ & $70 \mathrm{bcd}$ & $94 \mathrm{ab}$ & $100 \mathrm{a}$ & $76 b$ \\
\hline & $18 \% \mathrm{HzO}$ & $86 a b$ & $100 \mathrm{a}$ & $100 \mathrm{a}$ & $100 \mathrm{a}$ & $72 \mathrm{~b}$ \\
\hline & $14 \% \mathrm{HOO}$ & $98 a$ & $94 \mathrm{ab}$ & $98 \mathrm{a}$ & $14 \mathrm{~d}$ & $44 \mathrm{c}$ \\
\hline \multirow[t]{8}{*}{$20^{\circ} \mathrm{C} /$} & $48 \% \mathrm{H} O$ & $0 \mathrm{e}$ & $0 \mathrm{e}$ & 12 ef & $52 \mathrm{bc}$ & $0 \mathrm{e}$ \\
\hline & $43 \% \mathrm{H} O$ & $0 \mathrm{e}$ & $0 \mathrm{e}$ & $0 \mathrm{f}$ & $0 \mathrm{e}$ & $0 \mathrm{e}$ \\
\hline & $40 \% \mathrm{H} O$ & $0 \mathrm{e}$ & $0 \mathrm{e}$ & $0 \mathrm{f}$ & $0 \mathrm{e}$ & $0 \mathrm{e}$ \\
\hline & $34 \% \mathrm{HzO}$ & $0 \mathrm{e}$ & $0 \mathrm{e}$ & $0 \mathrm{f}$ & $0 \mathrm{e}$ & $0 \mathrm{e}$ \\
\hline & $30 \% \mathrm{H} O$ & 22 cde & $0 \mathrm{e}$ & $0 \mathrm{f}$ & $0 \mathrm{e}$ & $0 \mathrm{e}$ \\
\hline & $24 \% \mathrm{HzO}$ & $100 \mathrm{a}$ & $100 \mathrm{a}$ & $100 \mathrm{a}$ & $100 \mathrm{a}$ & $100 \mathrm{a}$ \\
\hline & $18 \% \mathrm{H} O$ & $100 \mathrm{a}$ & $100 \mathrm{a}$ & $100 \mathrm{a}$ & $100 \mathrm{a}$ & $100 \mathrm{a}$ \\
\hline & $14 \% \mathrm{H} O$ & $88 \mathrm{ab}$ & $84 \mathrm{abc}$ & $84 a b$ & $28 \mathrm{~cd}$ & $18 \mathrm{~cd}$ \\
\hline \multirow[t]{8}{*}{$10^{\circ} \mathrm{C} /$} & $48 \% \mathrm{HbO}$ & $0 \mathrm{e}$ & $0 \mathrm{e}$ & $0 \mathrm{f}$ & $0 \mathrm{e}$ & $0 \mathrm{e}$ \\
\hline & $43 \% \mathrm{H} O$ & $0 \mathrm{e}$ & $2 \mathrm{e}$ & $6 \mathrm{f}$ & $0 \mathrm{e}$ & $4 \mathrm{e}$ \\
\hline & $40 \% \mathrm{H} O$ & $6 \mathrm{de}$ & $0 \mathrm{e}$ & $0 \mathrm{f}$ & $0 \mathrm{e}$ & $0 \mathrm{e}$ \\
\hline & $34 \% \mathrm{HbO}$ & $4 \mathrm{de}$ & $2 \mathrm{e}$ & $0 \mathrm{f}$ & $0 \mathrm{e}$ & $0 \mathrm{e}$ \\
\hline & $30 \% \mathrm{HzO}$ & $90 a b$ & $42 \mathrm{~d}$ & $54 \mathrm{~cd}$ & $0 \mathrm{e}$ & $0 \mathrm{e}$ \\
\hline & $24 \% \mathrm{H} 2 \mathrm{O}$ & $100 \mathrm{a}$ & $100 \mathrm{a}$ & $100 \mathrm{a}$ & $100 \mathrm{a}$ & $100 \mathrm{a}$ \\
\hline & $18 \% \mathrm{H} O$ & $30 \mathrm{~cd}$ & $64 \mathrm{~cd}$ & $100 \mathrm{a}$ & $100 \mathrm{a}$ & $100 \mathrm{a}$ \\
\hline & $14 \% \mathrm{H} O$ & $58 \mathrm{bc}$ & $72 \mathrm{bc}$ & $70 \mathrm{bc}$ & $74 \mathrm{~b}$ & $88 \mathrm{~b}$ \\
\hline
\end{tabular}

1. Médias seguidas de mesma letra na coluna não diferem entre si, pelo teste de Tukey, ao nível de 5\% de probabilidade. 
Tabela 14. Incidência de Aspergillus sp. nas sementes de Myrciaria dubia: valores médios ${ }^{1}(\%)$ obtidos durante o armazenamento

\begin{tabular}{|c|c|c|c|c|c|c|}
\hline \multirow{2}{*}{\multicolumn{2}{|c|}{$\begin{array}{c}\text { Tratamentos (temperatura / } \\
\text { grau de umidade) }\end{array}$}} & \multicolumn{5}{|c|}{ Períodos de armazenamento (dias) } \\
\hline & & 35 & 70 & 140 & 210 & 280 \\
\hline \multirow[t]{8}{*}{$30^{\circ} \mathrm{C} /$} & $48 \% \mathrm{H} O$ & $0 \mathrm{~d}$ & $2 \mathrm{~cd}$ & $0 \mathrm{e}$ & $10 \mathrm{ef}$ & $0 \mathrm{f}$ \\
\hline & $43 \% \mathrm{HzO}$ & $0 \mathrm{~d}$ & $0 \mathrm{~d}$ & $0 \mathrm{e}$ & $0 \mathrm{~g}$ & $0 \mathrm{f}$ \\
\hline & $40 \% \mathrm{H} 2 \mathrm{O}$ & $0 \mathrm{~d}$ & $0 \mathrm{~d}$ & $0 \mathrm{e}$ & $0 \mathrm{~g}$ & $0 \mathrm{f}$ \\
\hline & $34 \% \mathrm{HbO}$ & $0 \mathrm{~d}$ & $0 \mathrm{~d}$ & $0 \mathrm{e}$ & $0 \mathrm{~g}$ & $0 \mathrm{f}$ \\
\hline & $30 \% \mathrm{HzO}$ & $0 \mathrm{~d}$ & $0 \mathrm{~d}$ & $0 \mathrm{e}$ & $4 \mathrm{fg}$ & $2 \mathrm{f}$ \\
\hline & $24 \% \mathrm{HbO}$ & $54 \mathrm{a}$ & $20 a b$ & $18 \mathrm{~d}$ & $86 \mathrm{abc}$ & $84 \mathrm{a}$ \\
\hline & $18 \% \mathrm{HbO}$ & $12 \mathrm{bcd}$ & $2 \mathrm{~cd}$ & $48 \mathrm{bc}$ & $56 \mathrm{~d}$ & 54 bc \\
\hline & $14 \% \mathrm{H}$ & $24 \mathrm{bc}$ & $24 \mathrm{ab}$ & $30 \mathrm{~cd}$ & $76 \mathrm{~cd}$ & $72 \mathrm{ab}$ \\
\hline \multirow[t]{8}{*}{$20^{\circ} \mathrm{C} /$} & $48 \% \mathrm{H}_{2} \mathrm{O}$ & $0 \mathrm{~d}$ & $0 \mathrm{~d}$ & $0 \mathrm{e}$ & $0 \mathrm{~g}$ & $0 \mathrm{f}$ \\
\hline & $43 \% \mathrm{HbO}$ & $0 \mathrm{~d}$ & $0 \mathrm{~d}$ & $0 \mathrm{e}$ & $0 \mathrm{~g}$ & $0 \mathrm{f}$ \\
\hline & $40 \% \mathrm{H}$ & $0 \mathrm{~d}$ & $0 \mathrm{~d}$ & $0 \mathrm{e}$ & $0 \mathrm{~g}$ & $0 \mathrm{f}$ \\
\hline & $34 \% \mathrm{H} 2 \mathrm{O}$ & $0 \mathrm{~d}$ & $2 \mathrm{~cd}$ & $0 \mathrm{e}$ & $0 \mathrm{~g}$ & $0 \mathrm{f}$ \\
\hline & $30 \% \mathrm{HbO}$ & $0 \mathrm{~d}$ & $0 \mathrm{~d}$ & $0 \mathrm{e}$ & $0 \mathrm{~g}$ & $0 \mathrm{f}$ \\
\hline & $24 \% \mathrm{H} 2 \mathrm{O}$ & $30 \mathrm{ab}$ & $14 \mathrm{bc}$ & $76 a$ & $92 \mathrm{ab}$ & $48 \mathrm{bcd}$ \\
\hline & $18 \% \mathrm{HbO}$ & $6 \mathrm{~cd}$ & $2 \mathrm{~cd}$ & $0 \mathrm{e}$ & $96 a$ & $2 \mathrm{f}$ \\
\hline & $14 \% \mathrm{HbO}$ & $12 \mathrm{bcd}$ & $48 \mathrm{a}$ & $30 \mathrm{~cd}$ & 82 bc & $10 \mathrm{ef}$ \\
\hline \multirow[t]{8}{*}{$10^{\circ} \mathrm{C} /$} & $48 \% \mathrm{HbO}$ & $0 \mathrm{~d}$ & $0 \mathrm{~d}$ & $0 \mathrm{e}$ & $0 \mathrm{~g}$ & $0 \mathrm{f}$ \\
\hline & $43 \% \mathrm{HbO}$ & $0 \mathrm{~d}$ & $0 \mathrm{~d}$ & $0 \mathrm{e}$ & $0 \mathrm{~g}$ & $0 \mathrm{f}$ \\
\hline & $40 \% \mathrm{H} O$ & $0 \mathrm{~d}$ & $0 \mathrm{~d}$ & $0 \mathrm{e}$ & $0 \mathrm{~g}$ & $0 \mathrm{f}$ \\
\hline & $34 \% \mathrm{H} 2 \mathrm{O}$ & $0 \mathrm{~d}$ & $0 \mathrm{~d}$ & $0 \mathrm{e}$ & $0 \mathrm{~g}$ & $0 \mathrm{f}$ \\
\hline & $30 \% \mathrm{H}_{2} \mathrm{O}$ & $2 \mathrm{~d}$ & $8 \mathrm{bcd}$ & $62 a b$ & $0 \mathrm{~g}$ & $0 \mathrm{f}$ \\
\hline & $24 \% \mathrm{H} 2 \mathrm{O}$ & $0 \mathrm{~d}$ & $0 \mathrm{~d}$ & $0 \mathrm{e}$ & 8 efg & $22 \mathrm{de}$ \\
\hline & $18 \% \mathrm{H}_{2} \mathrm{O}$ & $2 \mathrm{~d}$ & $6 \mathrm{bcd}$ & $0 \mathrm{e}$ & $20 \mathrm{e}$ & $0 \mathrm{f}$ \\
\hline & $14 \% \mathrm{H}$ & $20 \mathrm{bc}$ & $6 \mathrm{bcd}$ & $14 \mathrm{~d}$ & $20 \mathrm{e}$ & $36 \mathrm{~cd}$ \\
\hline
\end{tabular}

1. Médias seguidas de mesma letra na coluna não diferem entre si, pelo teste de Tukey, ao nível de 5\% de probabilidade. 


\section{CONCLUSÕES}

As sementes de Myrciaria dubia (H.B.K.) McVaugh têm a conservação favorecida pela associação do grau de umidade de $43 \%$ com a temperatura de armazenamento de $10^{\circ} \mathrm{C}$. Sob essa condição, a incidência de Fusarium sp. não prejudica o desempenho fisiológico das sementes. 


\section{REFERÊNCIAS BIBLIOGRÁFICAS}

ALVARADO V., M.A. Posibilidades del cultivo del camu camu en el Peru, Myrciaria dubia. Lima, 1969. 51p. Tesis (Ingeniero Agrónomo) - Pontificia Universidad Catolica del Peru.

ANDRADE, A.C.S.; CUNHA, R Grau crítico de umidade? Informativo do Comitê Técnico de Sementes Recalcitrantes, n.1, p.2-3, 1996.

ANDRADE, A.C.S.; MALAVASI, M.M.; COSTA, F.A. Conservação de palmiteiro (Euterpe edulis Mart.): efeito da temperatura de armazenamento e do grau de umidade das sementes. Revista Brasileira de Sementes, v.18, n.2, p.149-155, 1996.

ANDRADE, J.S. Curvas de maturação e características nutricionais do camu-camu Myrciaria dubia (H.B.K.) McVaugh cultivado em terra firme na Amazônia central brasileira. Campinas, 1991. 177p. Tese (Doutorado) - Faculdade de Engenharia de Alimentos, Universidade Estadual de Campinas.

ANDRADE, J.S.; ARAGÃO, C.G.; GALEAZZI, M.A.M.; FERREIRA, S.A.N. Changes in the concentration of total vitamin $\mathrm{C}$ during maturation and ripening of camu-camu (Myrciaria dubia (H.B.K.) McVaugh) fruits cultivated in the upland of brazilian central Amazon. Acta Horticulturae, n.370, p.177-180, 1995.

BACCHI, O. Estudos sobre a conservação de sementes. IX. Ingá. Bragantia, v.20, n.35, p.805-814, 1961.

BARBEDO, C.J. Armazenamento de sementes de Inga uruguensis Hook. \& Arn. Piracicaba, 1997. 71p. Tese (Doutorado) - Escola Superior de Agricultura "Luiz de Queiroz”, Universidade de São Paulo. 
BARBEDO, C.J.; KOHAMA, S.; MALUF, A.M.; BILIA, D.A.C. Germinação e armazenamento de diásporos de cerejeira (Eugenia involucrata DC. - Myrtaceae) em função do teor de água. Revista Brasileira de Sementes, v.20, n.1, p.184-188, 1998.

BARRUETO, L.P.; PEREIRA, I.P.; NEVES, M.A. Influência da maturação fisiológica e do período entre a coleta e o início do armazenamento, sobre a viabilidade da semente de seringueira (Hevea spp.). Turrialba, v.36, n.1, p.65-75, 1986.

BERJAK, P.; PAMMENTER, N.W. What ultrastructure has told us about recalcitrant seeds. Revista Brasileira de Fisiologia, v.12, p.22-55, 2000. Edição especial.

BILIA, D.A.C.; MARCOS FILHO, J.; NOVEMBRE, A.D.L.C. Conservação da qualidade fisiológica de sementes de Inga uruguensis Hook. et Arn. Revista Brasileira de Sementes, v.20, n.1, p.48-54, 1998.

BITENCOURT, L.F.; HOMECHIN, M. Avaliação da qualidade sanitária de sementes de guaçatonga (Casearia sylvestris Swartz - Flacourtiaceae) por três métodos de incubação. Revista Brasileira de Sementes, v.20, n.1, p.233-236, 1998.

BONNER, F.T. Storage of hardwood seeds. Forest Genetics Resources Information, n.7, p.10-17, 1978.

BOYCE, K.G. Report of the Seed Storage Committee 1986-1989. Seed Science and Technology, v.17, suppl.1, p.135-142, 1989.

BRASIL. Ministério da Agricultura e Reforma Agrária. Regras para análise de sementes. Brasília: CLAV; DNDV; SNAD/MA, 1992. 365p.

CALZADA B., J. 143 frutales nativos. La Molina: El Estudiante, 1980. 314p.

CALZADA B., J.; RODRIGUEZ R., J. Investigaciones sobre camu camu (Myrciaria paraensis Berg.). Iquitos: INIA, 1980. 15p.

CARDOSO, M.; ZINK, E.; BACCHI, O. Estudos sobre conservação de sementes de seringueira. Bragantia, v.25, p.35-40, 1966.

CASTAÑEDA, R.R. Frutas silvestres de Colombia. Bogotá: Author, 1961. 300p.

CHIN, H.F. Production and storage of recalcitrant seeds in the tropics. Acta Horticulturae, n.83, p.17-21, 1978.

CHIN, H.F. Recalcitrant seeds: a status report. Rome: IBPGR, 1988. 18p. 
CHRISTENSEN, C.M. Microflora and seed deterioration. In: ROBERTS, E.H. (Ed.) Viability of seeds. Syracuse: Syracuse University Press, 1972. cap.3, p.59-93.

COPELAND, L.O.; McDONALD, M.B. Principles of seed science and technology. 3.ed. New York: Chapman \& Hall, 1995. 409p.

CORBINEAU, F.; CÔME, D. Storage of recalcitrant seeds of four tropical species. Seed Science and Technology, v.16, p.97-103, 1988.

CROMARTY, A.S.; ELLIS, R.H.; ROBERTS, E.H. Desing of seed storage facilities for genetic conservation. Rome: IBPGR, 1985. 100p.

DELOUCHE, J.C. Physiology of seed storage. In: CORN AND SORGHUM RESEARCH CONFERENCE, 23., Washington, 1968. Proceedings. Washington: ASTA, 1968. p.83-90.

DELOUCHE, J.C.; BASKIN, C.C. Accelerated aging techniques for predicting the relative storability of seed lots. Seed Science and Technology, v.1, n.2, p.427-552, 1973.

ELLIS, R.H. The longevity of seeds. Hortscience, v.26, n.9, p.1119-1125, 1991.

ENCISO N., R.; VILLACHICA L., H. Produccion y manejo de plantas injertadas de camu camu (Myrciaria dubia) en vivero. Lima: INIA, 1993. 20p. (Informe Tecnico, 25).

EVANS, H. Results of some experiments on the preservation of cacao seed in viable condition. Tropical Agriculture, v.26, p.48-55, 1950.

FARRANT, J.M.; PAMMENTER, N.W.; BERJAK, P. Germination-associated events and the desiccation sensitivity of recalcitrant seeds - a study on three unrelated species. Planta, v.178, n.2, p.189-198, 1989.

FERRAZ, I.D.K.; SAMPAIO, P.T.B. Métodos simples de armazenamento das sementes de andiroba (Carapa guianensis Aubl. e Carapa procera D.C. - Meliaceae). Acta Amazonica, v.26, n.3, p.137-144, 1996.

FERREIRA, P.V. Estatística experimental aplicada à Agronomia. Maceió: EDUFAL, 1991. 437p.

FERREIRA, S.A.N. Camu-camu. Informativo SBF, v.5, n.2, p.11-12, 1986. 
FERREIRA, S.A.N.; GENTIL, D.F.O. Propagação assexuada do camu-camu (Myrciaria dubia) através de enxertias do tipo garfagem. Acta Amazonica, v.27, n.3, p.163-168, 1997.

FIGUEIREDO, S.F.L. Conservação da viabilidade da semente de cacau. IV. Efeitos de fungicidas e peletização. Revista Theobroma, v.16, n.4, p.173-188, 1986.

FU, J.R.; ZHANG, B.Z.; WANG, X.P.; QIAO, Y.P.; HUANG, X.L. Physiological studies on desiccation, wet storage and cryopreservation of recalcitrant seeds of three fruit species and their excised embryonic axes. Seed Science and Technology, v.18, p.743-754, 1990.

GENTIL, D.F.O. Conservação de sementes do cafeeiro: resultados discordantes ou complementares? Bragantia, v.60, n.3, p.149-154, 2001.

GENTIL, D.F.O.; FERREIRA, S.A.N. Armazenamento de sementes de camu-camu (Myrciaria dubia (H. B. K.) McVaugh). In: SEMINÁRIO ANUAL DE INICIAÇÃO CIENTÍFICA, 4., Manaus, 1991. Resumos. Manaus: UA; INPA, 1991. p.10.

GENTIL, D.F.O.; FERREIRA, S.A.N. Tolerância à dessecação e viabilidade de sementes de camu-camu. Revista Brasileira de Fruticultura, v.22, n.2, p.264-267, 2000.

GOLDBACH, H. Imbibed storage of Melicoccus bijugatus and Eugenia brasiliensis (E. dombeyi) using abscisic acid as a germination inhibitor. Seed Science and Technology, v.7, p.403-406, 1979.

GUTIERREZ R., A. Especies frutales nativas de la selva del Peru: estudio botânico e de propagación de semillas. Lima, 1969. Tesis (Ingeniero Agrónomo) - Universidad Nacional Agraria.

HARRINGTON, J.F. Seed storage and longevity. In: KOZLOWSKI, T.T. (Ed.). Seed biology: insects, and seed collection, storage, testing, and certification. New York: Academic Press, 1972. v.3, cap.3, p.145-245. (Physiological Ecology: A Series of Monographs, Texts, and Treatises).

HOEKSTRA, F.A.; GOLOVINA, E.A.; BUITINK, J. Mechanisms of plant desccation tolerance. Trends in Plant Science, v.6, n.9, p.431-438, 2001. 
HONG, T.D.; ELLIS, R.H. Optimum air-dry seed storage environments for arabica coffee. Seed Science and Technology, v.20, p.547-560, 1992.

HONG, T.D.; ELLIS, R.H. A protocol to determine seed storage behaviour. Rome: IPGRI, 1996. 62p. (IPGRI. Technical Bulletin, 1).

HONG, T.D.; LENINGTON, S.; ELLIS, R.H. Seed storage behaviour: a compendium. Rome: IPGRI, 1996. 101p. (Handbook for Genebanks, 4).

HOR, Y.L.; CHIN, H.F.; KARIM, M.Z. The effect of seed moisture and storage temperature on the storability of cocoa (Theobroma cacao) seeds. Seed Science and Technology, v.12, n.2, p.415-420, 1984.

IBRAHIM, A.E.; ROBERTS, E.H. Viability of lettuce seeds. I. Survival in hermetic storage. Journal of Experimental Botany, v.34, n.142, p.620-630, 1983.

IBRAHIM, A.E.; ROBERTS, E.H.; MURDOCH, A.J. Viability of lettuce seeds. II. Survival and oxygen uptake in osmotically controlled storage. Journal of Experimental Botany, v.34, n.142, p.631-640, 1983.

KEEL, S.H.K.; PRANCE, G.T. Studies of the vegetation of a white-sand black-water igapó (Rio Negro, Brazil). Acta Amazonica, v.9, n.4, p.645-655, 1979.

KING, M.W.; ROBERTS, E.H. The storage of recalcitrant seeds: achievements and possible approaches. Rome: IBPGR, 1979. 96p.

KING, M.W.; ROBERTS, E.H. The imbibed storage of cocoa (Theobroma cacao) seeds. Seed Science and Technology, v.10, n.3, p.535-540, 1982.

MAGUIRE, J.D. Speed of germination: aid in selection and evaluation for seedling emergence and vigour. Crop Science, v.2, n.2, p.176-177, 1962.

MARCOS FILHO, J. Testes de vigor: importância e utilização. In: KRZYZANOWSKI, F.C.; VIEIRA, R.D.; FRANÇA NETO, J.B. Vigor de sementes: conceitos e testes. Londrina: ABRATES, 1999. cap.1, p.1-21.

MARTINS, C.C.; NAKAGAWA, J.; BOVI, M.L.A. Tolerância à dessecação de sementes de palmito-vermelho (Euterpe espiritosantensis Fernandes). Revista Brasileira de Botânica, v.22, n.3, p.391-396, 1999.

McVAUGH, R. Flora of Peru IV - 2. Field Museum of Natural History - Botany, v.13, p.780-781, 1958. 
McVAUGH, R. Tropical american myrtaceae. Fieldiana - Botany, v.29, n.8, p.393532, 1963.

McVAUGH, R. Botany of the Guyana highland. Party VIII. Memoirs of the New York Botanical Garden, v.18, n.2, p.55-286, 1969.

MENTEN, J.O.M. I semana de atualização em patologia de sementes. Piracicaba: FEALQ, 1988. 76p.

MERA, P.A.S. Camu-camu Myrciaria dubia (H.B.K.) McVaugh. In: PRANCE, G.T.

Botânica econômica de algumas espécies amazônicas. Manaus: INPA; FUA, 1987. s.p.

MUMFORD, P.M.; BRETT, A.C. Conservation of cacao seed. Tropical Agriculture, v.59, n.4, p.306-310, 1982.

NAKAGAWA, J. Testes de vigor baseados na avaliação das plântulas. In: VIEIRA, R.D.; CARVALHO, N.M. Testes de vigor em sementes. Jaboticabal: FUNEP, 1994. p. 49-85.

PAMMENTER, N.W.; BERJAK, P. A review of recalcitrant seed physiology in relation to desiccation-tolerance mechanisms. Seed Science Research, v.9, n.1, p.13-37, 1999.

PETERS, C.M.; VASQUEZ, A. Estudios ecológicos de camu camu (Myrciaria dubia).

I. Producción de frutos en poblaciones naturales. Acta Amazonica, v.16/17, n. único, p.161-174, 1987.

PICÓN B., C.P.; FLOR B., F.D.; TRUEBA, C.P. Descriptores de camu camu. Lima: INIPA, 1987. 55p. (Informe Tecnico, 8).

POPINIGIS, F. Fisiologia da semente. Brasília: AGIPLAN, 1977. 289p.

PROBERT, R.; SMITH, R. Seed viability and the prediction of longevity. /Apresentado ao Seed Conservation Training Course, Jaboticabal, SP, 1996/.

QUEIROZ, M.H.; CAVALCANTE, M.D.T.H. Efeito do dessecamento das sementes de palmiteiro na germinação e no armazenamento. Revista Brasileira de Sementes, v.8, n.3, p.121-125, 1986. 
ROBERTS, E.H. Storage environment and the control of viability. In: ROBERTS, E.H. (Ed.). Viability of seeds. Syracuse: Syracuse University Press, 1972. cap.2, p.1458.

ROBERTS, E.H. Predicting the storage life of seeds. Seed Science and Technology, v.1, p.499-514, 1973.

ROBERTS, E.H.; ELLIS, R.H. Water and seed survival. Annals of Botany, v.63, n.1, p.39-52, 1989.

ROBERTS, E.H.; KING, M.W. The characteristics of recalcitrant seeds. In: CHIN, H.F.; ROBERTS, E.H. (Ed.). Recalcitrant crop seeds. Kuala Lumpur: Tropical Press, 1980. cap.1, p.1-5.

SANTANA, S.C. Propagação vegetativa, por meio de estaquia e enxertia com diferentes porta-enxertos de Myrtaceae, para camu-camu (Myrciaria dubia (H.B.K.) McVaugh). Manaus, 1998. 89p. Dissertação (Mestrado) - Instituto Nacional de Pesquisas da Amazônia, Universidade do Amazonas.

SILVA, C.T.C.; ANDRADE, J.S. Postharvest modifications in camu-camu fruit (Myrciaria dubia McVaugh) in response to stage of maturation and modified atmosphere. Acta Horticulturae, n.452, p.23-26, 1997.

SIMÃO, S. Estudo do poder germinativo da semente de manga. Anais da Escola Superior de Agricultura 'Luiz de Queiroz”, v.16, p.289-297, 1959.

SPALDING, D.H.; KNIGHT JR., R.J.; REEDER, W.F. Storage of avocado seeds. Proceeding of the Florida State for Horticultural Society, v.89, n.2-4, p.257-258, 1976.

SPOSITO, M.B.; GENTIL, D.F.O.; ARAÚJO, P.S.R. Influência da temperatura no processo germinativo de sementes de camu-camu. In: CONGRESSO BRASILEIRO DE FRUTICULTURA, 15., Poços de Caldas, 1998. Resumos. Lavras: UFLA, 1998. p.765.

SUGUINO, E. Propagação vegetativa do camu-camu (Myrciaria dubia (H.B.K.) McVaugh) por meio da garfagem em diferentes porta-enxertos da família Myrtaceae. Piracicaba, 2002. 63p. Dissertação (Mestrado) - Escola Superior de Agricultura “Luiz de Queiroz”, Universidade de São Paulo. 
SUN, W.Q.; LEOPOLD, A.C. Cytoplasmic vitrification and survival of anhydrobiotic organisms. Comparative Biochemistry and Physiology, v.117A, n.3, p.327-333, 1997.

TOLEDO, F.F.; MARCOS FILHO, J. Manual das sementes: tecnologia da produção. São Paulo: Agronômica Ceres, 1977. 224p.

VILLACHICA L., H. El cultivo del camu camu (Myrciaria dubia H.B.K. McVaugh) en la Amazonia peruana. Caracas: TCA, 1996. 95p.

VILLACHICA L., H.; LABARTE F.; J. Camu camu: descripción del producto y situación actual del cultivo. Prompex, p.73-87, 1998.

VILLACHICA L., H.; CARVALHO, J.E.U.; MÜLLER, C.H.; DIAZ S., C.; ALMANZA, M. Frutales y hortalizas promisorios de la Amazonia. Lima: TCA, 1995. 367p.

VILLALOBOS, R.; HERRERA, J.; MORA-URPÍ, J. Germinación de la semilla de pejibaye (Bactris gasipaes). III. Efecto del contenido de agua y de las condiciones de almacenamiento. Agronomía Costarricense, v.16, n.1, p.69-76, 1992.

WALTERS, C. Levels of recalcitrance in seeds. Revista Brasileira de Fisiologia, v.12, p.7-21, 2000. Edição especial.

XIA, Q.H.; CHEN, R.Z.; FU, J.R. Moist storage of lychee (Litchi chinensis Sonn.) and logan (Euphoria longan Steud.) seeds. Seed Science and Technology, v.20, n.2, p.269-279, 1992.

ZINK, E.; ROCHELLE, L.A. Estudos sobre a conservação de sementes. XI. Cacau. Bragantia, v.23, n.11, p.111-116, 1964.

ZONTA, E.P.; MACHADO, A.A. Programa SANEST: sistema de análise estatística para microcomputadores (software). Pelotas: UFPEL, 1984. 